\title{
Certificateless KEM and Hybrid Signcryption Schemes Revisited
}

\author{
S. Sharmila Deva Selvi, S. Sree Vivek * and C. Pandu Rangan ${ }^{\star}$ \\ Theoretical Computer Science Lab, \\ Department of Computer Science and Engineering, \\ Indian Institute of Technology Madras, India. \\ \{sharmila, svivek, prangan\}@cse.iitm.ac.in
}

\begin{abstract}
Often authentication and confidentiality are required as simultaneous key requirements in many cryptographic applications. The cryptographic primitive called signcryption effectively implements the same and while most of the public key based systems are appropriate for small messages, hybrid encryption (KEM-DEM) provides an efficient and practical way to securely communicate very large messages. Recently, Lippold et al. [13] proposed a certificateless KEM in the standard model and the first certificateless hybrid signcryption scheme was proposed by Fagen Li et al. [15]. The concept of certificateless hybrid signcryption has evolved by combining the ideas of signcryption based on tagKEM and certificateless cryptography. In this paper, we show that [13] is not Type-I CCA secure and [15] is existentially forgeable. We also propose an improved certificateless hybrid signcryption scheme and formally prove the security of the improved scheme against both adaptive chosen ciphertext attack and existential forgery in the appropriate security models for certificateless hybrid signcryption.
\end{abstract}

Keywords. Certificateless Cryptography, Signcryption, Cryptanalysis, Hybrid Signcryption, Tag-KEM, Bilinear Pairing, Provable Security, Random Oracle Model.

\section{Introduction}

In 1984, Shamir [21] introduced the concept of identity based cryptography (IBC) and proposed the first identity based signature scheme. The idea of identity based cryptography is to enable a user to use any arbitrary string, that uniquely identifies him as his public key. Identity based cryptography serves as an efficient alternative to Public Key Infrastructure (PKI) because no certificate is needed to validate the public key of a user. Identity based cryptosystem makes use of a trusted third party, the private key generator (PKG), who is in possession of a master secret key which is used to derive the private key of any user in the system. Thus the private key of all the user in the system is known to the PKG, since it was generated by him. This is an inherent issue in IBC and is called as the key escrow problem. Certificateless Cryptography (CLC) was introduced by Al-Riyami at al. [1] to reduce the trust level of KGC (The trusted third party in CLC is the Key Generation Center) and thus to find an effective remedy to the key escrow problem in IBC. This can be achieved by splitting the private key into two parts; one is generated by the KGC and is known as the partial private key, other one is a user selected secret value. Any cryptographic operations can only be done with both these private key components or a combination of both. The public key is no longer the identity of the user in CLC but it is derived from the partial private key and the secret value of the corresponding user. The main challenge in building a CLC is to build a system that can resist two types of attacks namely Type-I and Type-II attacks (described later in the paper).

All the Certificateless Key Encapsulation Mechanism (CL-KEM) schemes [6], [14] till date are generic constructions, i.e. they combine a public key based encryption scheme and an identity based KEM and thus very in-efficient. Lippold et al. [13] proposed the first direct construction for a CCA secure CL-KEM in the standard model.

Simultaneous confidentiality and authentication of messages are often required for secure and authentic message transmission over an insecure channel like internet. Signcryption is the cryptographic primitive that offers both these properties with a very low cost when compared to encrypting and signing a message

\footnotetext{
* Work supported by Project No. CSE/05-06/076/DITX/CPAN on Protocols for Secure Communication and Computation sponsored by Department of Information Technology, Government of India
} 
independently. Zheng [25] introduced the concept of signcryption in 1997 and many signcryption schemes were proposed till date $[18,17,9,7,16,5,8,4,20]$, to name a few. Baek et al. in [3] gave the formal security model for signcryption and proved the security of [25] in the model. There are two different ways to construct signcryption schemes, one is public key signcryption and other is hybrid signcryption. In a public key signcryption scheme both encryption and signature are in public key setting. A few examples for this type of construct are schemes by An et al. [2], Malone-Mao [19] and Dodis et al.[12]. In a Hybrid signcryption scheme, the signature is in public key setting and encryption is in symmetric key setting, here an one-time secret key which is used in the symmetric key encryption of the message is encrypted by a public key encryption algorithm. The formal security model for a hybrid signcryption scheme was given by Dent [11] and Bjørstad [23]. Generation of secret key and encrypting it using a public key encryption scheme is called key encapsulation mechanism (KEM) and encrypting the message with the secret key and a symmetric key encryption scheme is called as data encryption mechanism (DEM). The definitions and formal treatment of $\mathrm{KEM} / \mathrm{DEM}$ can be found in [10] and [22].

Our Contribution: Our contribution in this paper is three fold. First, we show that the CL-KEM in [13] is not CCA secure. To the best of our knowledge, there exists only one certificateless hybrid signcryption scheme (CLSC-TKEM) by Fagen Li et al. [15]. Fagen Li et al. have proposed the first CLSC-TKEM and proposed the security model for it. Next, we show that the scheme proposed in [15] is existentially forgeable. Finally, we propose an improved certificateless hybrid signcryption scheme and prove its security in the random oracle model.

\section{Preliminaries}

\subsection{Bilinear Pairing}

Let $\mathbb{G}_{1}$ be an additive cyclic group generated by $P$, with prime order $q$, and $\mathbb{G}_{2}$ be a multiplicative cyclic group of the same order. A bilinear pairing is a map $\hat{e}: \mathbb{G}_{1} \times \mathbb{G}_{1} \rightarrow \mathbb{G}_{2}$ with the following properties.

- Bilinearity. For all $P, Q, R \in \mathbb{G}_{1}$,

- $\hat{e}(P+Q, R)=\hat{e}(P, R) \hat{e}(Q, R)$

- $\hat{e}(P, Q+R)=\hat{e}(P, Q) \hat{e}(P, R)$

- $\hat{e}(a P, b Q)=\hat{e}(P, Q)^{a b}\left[\right.$ Where $\left.a, b \in \in_{R} \mathbb{Z}_{q}^{*}\right]$

- Non-Degeneracy. There exist $P, Q \in \mathbb{G}_{1}$ such that $\hat{e}(P, Q) \neq I_{\mathbb{G}_{2}}$, where $I_{\mathbb{G}_{2}}$ is the identity element of $\mathbb{G}_{2}$.

- Computability. There exists an efficient algorithm to compute $\hat{e}(P, Q)$ for all $P, Q \in \mathbb{G}_{1}$.

\subsection{Computational Assumptions}

In this section, we review the computational assumptions relevant to the protocol we propose.

Computation Diffie-Hellman Problem (CDH) Given $(P, a P, b P) \in \mathbb{G}_{1}^{3}$ for unknown $a, b \in \mathbb{Z}_{q}^{*}$, the $\mathrm{CDH}$ problem in $\mathbb{G}_{1}$ is to compute $a b P$.

Definition. The advantage of any probabilistic polynomial time algorithm $\mathcal{A}$ in solving the CDH problem in $\mathbb{G}_{1}$ is defined as:

$$
A d v_{\mathcal{A}}^{C D H}=\operatorname{Pr}\left[\mathcal{A}(P, a P, b P)=a b P \mid a, b \in \mathbb{Z}_{q}^{*}\right]
$$

The $C D H$ Assumption is that, for any probabilistic polynomial time algorithm $\mathcal{A}$, the advantage $A d v_{\mathcal{A}}^{C D H}$ is negligibly small.

Computational Bilinear Diffie-Hellman Problem (CBDH) Given $(P, a P, b P, c P) \in \mathbb{G}_{1}^{4}$ for unknown $a, b, c \in \mathbb{Z}_{q}^{*}$, the CBDH problem in $\mathbb{G}_{1}$ is to compute $\hat{e}(P, P)^{a b c}$.

Definition. The advantage of any probabilistic polynomial time algorithm $\mathcal{A}$ in solving the CBDH problem in $\mathbb{G}_{1}$ is defined as:

$$
\left.A d v_{\mathcal{A}}^{C B D H}=\operatorname{Pr}\left[\mathcal{A}(P, a P, b P, c P)=\hat{e}(P, P)^{a b c}\right)=1 \mid a, b, c \in \mathbb{Z}_{q}^{*}\right]
$$

The $C B D H$ Assumption is that, for any probabilistic polynomial time algorithm $\mathcal{A}$, the advantage $A d v_{\mathcal{A}}^{C B D H}$ is negligibly small. 


\subsection{Certificateless Signcryption Tag-KEM (CLSC-TKEM)}

A generic Certificateless Signcryption Tag-KEM scheme consists of the following seven probabilistic polynomial time algorithms:

- Setup $(\kappa)$. Given a security parameter $\kappa$, the Key Generation Center (KGC) generates the public parameters params and master secret key $m s k$ of the system.

- Extract Partial Private Key $\left(I D_{A}\right)$. Given an identity $I D_{A} \in_{R}\{0,1\}^{*}$ of a user $A$ as input, the KGC computes the corresponding partial private key $D_{A}$ and gives it to $A$ in a secure way.

- Generate User Key $\left(I D_{A}\right)$. Given an identity $\left(I D_{A}\right)$ as input, this algorithm outputs a secret value $x_{A}$ and a public key $P K_{A}$. This algorithm is executed by the user $A$ to obtain his secret value which is used to generate his full private key and the corresponding public key which is published without certification.

- Set Private Key $\left(D_{A}, x_{A}\right)$. The input to this algorithm are the partial private key $D_{A}$ and the secret value $x_{A}$ of a user $A$. This algorithm is executed by the user $A$ to generate his full private key $S_{A}$.

- $\operatorname{Sym}\left(I D_{A}, P K_{A}, S_{A}, I D_{B}, P K_{B}\right)$. This is a symmetric key generation algorithm which takes the sender's identity $I D_{A}$, public key $P K_{A}$, private key $S_{A}$, the receiver's identity $I D_{B}$ and public key $P K_{B}$ as input. It is executed by the sender $A$ in order to obtain the symmetric key $K$ and an internal state information $\omega$, which is not known to $B$.

- Encap $(\omega, \tau)$. This is the key encapsulation algorithm which takes a state information $\omega$, an arbitrary $\operatorname{tag} \tau$, the sender's identity $I D_{A}$, public key $P K_{A}$ and private key $S_{A}$ as input. This algorithm is executed by the sender $A$ in order to obtain the encapsulation $\psi$. The values $\tau$ and $\psi$ are sent to $B$.

- Decap $\left(\psi, \tau, I D_{A}, P K_{A}, I D_{B}, P K_{B}, S_{B}\right)$. In order to obtain the encapsulated key $K$, the receiver $B$ runs this algorithm. The input to this algorithm are the encapsulation $\psi$, a tag $\tau$, the sender's identity $I D_{A}$, public key $P K_{A}$, the receiver's identity $I D_{B}$, public key $P K_{B}$ and private key $S_{B}$. The output of this algorithm is a key $K$ or invalid with respect to the validity of $\psi$.

The consistency constraint we require is, if $(K, \omega)=\operatorname{Sym}\left(I D_{A}, P K_{A}, S_{A}, I D_{B}, P K_{B}\right)$ and $\psi=\operatorname{Encap}(\omega, \tau)$, then $K=\operatorname{Decap}\left(\psi, \tau, I D_{A}, P K_{A}, I D_{B}, P K_{B}, S_{B}\right)$.

\subsection{Security Model for CLSC-TKEM}

The security notions for certificateless signcryption scheme was first formalized by Barbosa et al. in [4]. A CLSC scheme should satisfy indistinguishability against adaptive chosen ciphertext and identity attacks and existential unforgeability against adaptive chosen message and identity attacks. We describe below the security models to prove the confidentiality and unforgeability of a CLSC-TKEM scheme. These are the strongest security notions for this problem.

Confidentiality To prove the confidentiality of CLSC-TKEM scheme, we consider two games "IND-CLSCTKEM-CCA2-I" and "IND-CLSC-TKEM-CCA2-II". A Type-I adversary $\mathcal{A}_{I}$ interacts with the challenger $\mathcal{C}$ in the IND-CLSC-TKEM-CCA2-I game and a Type-II adversary $\mathcal{A}_{I I}$ interacts with the challenger $\mathcal{C}$ in the IND-CLSC-TKEM-CCA2-II game. A CLSC-TKEM scheme is indistinguishable against adaptive chosen ciphertext attacks (IND-CLSC-TKEM-CCA2), if no polynomially bounded adversaries $\mathcal{A}_{I}$ and $\mathcal{A}_{I I}$ have nonnegligible advantage in both IND-CLSC-TKEM-CCA2-I and IND-CLSC-TKEM-CCA2-II games between $\mathcal{C}$ and $\mathcal{A}_{I}, \mathcal{A}_{I I}$ respectively:

IND-CLSC-TKEM-CCA2-I: The following is the interactive game between $\mathcal{C}$ and $\mathcal{A}_{I}$.

Setup: The challenger $\mathcal{C}$ runs this algorithm to generate the master public and private keys, params and $m s k$ respectively. $\mathcal{C}$ gives params to $\mathcal{A}_{I}$ and keeps the master private key $m s k$ secret from $\mathcal{A}_{I}$.

Phase 1: $\mathcal{A}_{I}$ performs a series of queries in an adaptive fashion in this phase. The queries allowed are given below:

- Extract Partial Private Key queries: $\mathcal{A}$ chooses an identity $I D_{i}$ and gives it to $\mathcal{C}$. $\mathcal{C}$ computes the corresponding partial private key $D_{i}$ and sends it to $\mathcal{A}_{I}$.

- Extract Private Key queries: $\mathcal{A}_{I}$ produces an identity $I D_{i}$ and requests the corresponding full private key. If $I D_{i}$ 's public key has not been replaced then $\mathcal{C}$ responds with the full private key $S_{i}$. If $\mathcal{A}_{I}$ has already replaced $I D_{i}$ 's public key, then $\mathcal{C}$ does not provide the corresponding private key to $\mathcal{A}_{I}$. 
- Request Public Key queries: $\mathcal{A}_{I}$ produces an identity $I D_{i}$ to $\mathcal{C}$ and requests $I D_{i}$ 's public key. $\mathcal{C}$ responds by returning the public key $P K_{i}$ for the user $I D_{i}$. (First by choosing a secret value if necessary).

- Replace Public Key queries: $\mathcal{A}_{I}$ can repeatedly replace the public key $P K_{i}$ corresponding to the user identity $I D_{i}$ with any value $P K_{i}^{\prime}$ of $\mathcal{A}_{I}$ 's choice. The current value of the user's public key is used by $\mathcal{C}$ in any computations or responses to $\mathcal{A}_{I}$ 's queries.

- Symmetric Key Generation queries: $\mathcal{A}_{I}$ produces a sender's identity $I D_{A}$, public key $P K_{A}$, the receiver's identity $I D_{B}$ and public key $P K_{B}$ to $\mathcal{C}$. The private key of the sender $S_{A}$ is obtained from the corresponding list maintained by $\mathcal{C}$. $\mathcal{C}$ computes the symmetric key $K$ and an internal state information $\omega$, stores and keeps $\omega$ secret from the view of $\mathcal{A}_{I}$ and sends the symmetric key $K$ to $\mathcal{A}_{I}$. It is to be noted that $\mathcal{C}$ may not be aware of the corresponding private key if the public key of $I D_{A}$ is replaced. In this case $\mathcal{A}_{I}$ provides the private key of $I D_{A}$ to $\mathcal{C}$.

- Key Encapsulation queries: $\mathcal{A}_{I}$ produces an arbitrary tag $\tau$, the sender's identity $I D_{A}$ and public key $P K_{A}$, The private key of the sender $S_{A}$ is known to $\mathcal{C}$. $\mathcal{C}$ checks whether a corresponding $\omega$ value is stored previously. If $\omega$ exists then $\mathcal{C}$ computes the encapsulation $\psi$ with $\omega$ and $\tau$ and deletes $\omega$, else returns invalid.

- Key Decapsulation queries: $\mathcal{A}_{I}$ produces an encapsulation $\psi$, a tag $\tau$, the sender's identity $I D_{A}$, public key $P K_{A}$, the receiver's identity $I D_{B}$ and public key $P K_{B}$. The private key of the receiver $S_{B}$ is obtained from the corresponding list maintained by $\mathcal{C}$. $\mathcal{C}$ returns the key $K$ or invalid with respect to the validity of $\psi$. It is to be noted that $\mathcal{C}$ may not be aware of the corresponding private key if the public key of $I D_{B}$ is replaced. In this case $\mathcal{A}_{I}$ provides the private key of $I D_{B}$ to $\mathcal{C}$.

Challenge: At the end of Phase 1 (which is decided by $\mathcal{A}_{I}$ ), $\mathcal{A}_{I}$ sends to $\mathcal{C}$, a sender identity $I D_{A^{*}}$ and a receiver identity $I D_{B^{*}}$ on which $\mathcal{A}_{I}$ wishes to be challenged. Here, the private key of the receiver $I D_{B^{*}}$ was not queried in Phase 1. Now, $\mathcal{C}$ computes $\left\langle K_{1}, \omega^{*}\right\rangle$ using $\operatorname{Sym}\left(I D_{A}, P K_{A}, S_{A}, I D_{B}, P K_{B}\right)$ and chooses $K_{0} \in_{R} \mathcal{K}$, where $\mathcal{K}$ is the key space of the CLSC-TKEM scheme. Now $\mathcal{C}$ chooses a bit $\delta \in_{R}\{0,1\}$ and sends $K_{\delta}$ to $\mathcal{A}_{I}$. When $\mathcal{A}_{I}$ receives $K_{\delta}$, it generates an arbitrary tag $\tau^{*}$ and sends it to $\mathcal{C}$. $\mathcal{C}$ computes the challenge encapsulation $\psi^{*}$ with $\omega^{*}$ and $\tau^{*}$ and sends $\psi^{*}$ to $\mathcal{A}_{I}$.

Phase 2: $\mathcal{A}_{I}$ can perform polynomially bounded number of queries adaptively again as in Phase 1 but it cannot make a partial private key extraction query on $I D_{B^{*}}$ or cannot query for the decapsulation of $\psi^{*}$. If the public key of $I D_{B^{*}}$ is replaced after the Challenge, $\mathcal{A}_{I}$ can ask for the decapsulation of $\psi^{*}$.

Guess: $\mathcal{A}_{I}$ outputs a bit $\delta^{\prime}$ and wins the game if $\delta^{\prime}=\delta$.

The advantage of $\mathcal{A}_{I}$ is defined as $A d v^{I N D-C L S C-T K E M-C C A 2-I}\left(\mathcal{A}_{I}\right)=\left|2 \operatorname{Pr}\left[\delta^{\prime}=\delta\right]-1\right|$, where $\operatorname{Pr}\left[\delta^{\prime}=\delta\right]$ denotes the probability that $\delta^{\prime}=\delta$.

IND-CLSC-TKEM-CCA2-II: The following is the interactive game between $\mathcal{C}$ and $\mathcal{A}_{I I}$.

Setup: The challenger $\mathcal{C}$ runs this algorithm to generate the master public and private keys, params and $m s k$ respectively. $\mathcal{C}$ gives both params and $m s k$ to $\mathcal{A}_{I I}$.

Phase 1: $\mathcal{A}_{I I}$ performs a series of queries in an adaptive fashion in this phase. The queries allowed are similar to that of the IND-CLSC-TKEM-CCA2-I game except that Extract Partial Private Key queries: is excluded because $\mathcal{A}_{I I}$ can generate it on need basis as it knows $m s k$.

Challenge: At the end of Phase 1 (which is decided by $\mathcal{A}_{I I}$ ), $\mathcal{A}_{I I}$ sends to $\mathcal{C}$, a sender identity $I D_{A^{*}}$ and a receiver identity $I D_{B^{*}}$ on which $\mathcal{A}_{I I}$ wishes to be challenged. Here, the full private key of the receiver $I D_{B^{*}}$ was not queried in Phase 1. Now, $\mathcal{C}$ computes $\left\langle K_{1}, \omega^{*}\right\rangle$ using $\operatorname{Sym}\left(I D_{A}, P K_{A}, S_{A}, I D_{B}, P K_{B}\right)$ and chooses $K_{0} \in_{R} \mathcal{K}$, where $\mathcal{K}$ is the key space of the CLSC-TKEM scheme. Now $\mathcal{C}$ chooses a bit $\delta \in_{R}\{0,1\}$ and sends $K_{\delta}$ to $\mathcal{A}_{I I}$. When $\mathcal{A}_{I I}$ receives $K_{\delta}$, it generates an arbitrary tag $\tau^{*}$ and sends it to $\mathcal{C}$. $\mathcal{C}$ computes the challenge encapsulation $\psi^{*}$ with $\omega^{*}$ and $\tau^{*}$ and sends $\psi^{*}$ to $\mathcal{A}_{I I}$.

Phase 2: $\mathcal{A}_{I I}$ can perform ploynomially bounded number of queries adaptively again as in Phase 1 but it cannot make a partial private key extraction query on $I D_{B^{*}}$ or cannot query for the decapsulation of $\psi^{*}$. If the public key of $I D_{B^{*}}$ is replaced after the Challenge, $\mathcal{A}_{I}$ can ask for the decapsulation of $\psi^{*}$.

Guess: $\mathcal{A}_{I I}$ outputs a bit $b^{\prime}$ and wins the game if $b^{\prime}=b$.

The advantage of $\mathcal{A}_{I I}$ is defined as $A d v^{I N D-C L S C-T K E M-C C A 2-I I}\left(\mathcal{A}_{I I}\right)=\left|2 \operatorname{Pr}\left[\delta^{\prime}=\delta\right]-1\right|$, where $\operatorname{Pr}\left[\delta^{\prime}=\right.$ $\delta$ ] denotes the probability that $\delta^{\prime}=\delta$. 
Existential Unforgeability To prove the existential unforgeability of CLSC-TKEM scheme, we consider two games "EUF-CLSC-TKEM-CMA-I" and "EUF-CLSC-TKEM-CMA-II". A Type-I forger $\mathcal{F}_{I}$ interacts with the challenger $\mathcal{C}$ in the EUF-CLSC-TKEM-CMA-I game and a Type-II forger $\mathcal{F}_{I I}$ interacts with $\mathcal{C}$ in the EUF-CLSC-TKEM-CMA-II game. A CLSC-TKEM scheme is existentially unforgeable against adaptive chosen message attack (EUF-CLSC-TKEM-CMA), if no polynomially bounded forgers $\mathcal{F}_{I}$ and $\mathcal{F}_{I I}$ have nonnegligible advantage in both EUF-CLSC-TKEM-CMA-I and EUF-CLSC-TKEM-CMA-II games between $\mathcal{C}$ and $\mathcal{F}_{I}, \mathcal{F}_{I I}$ respectively:

$\boldsymbol{E} \boldsymbol{U} \boldsymbol{F}-\boldsymbol{C L S C}$ - TKEM-CMA-I: The following is the interactive game between $\mathcal{C}$ and $\mathcal{F}_{I}$ :

Setup: The challenger $\mathcal{C}$ runs this algorithm to generate the master public and private keys, params and msk respectively. $\mathcal{C}$ gives params to $\mathcal{F}_{I}$ and keeps the master private key $m s k$ secret from $\mathcal{F}_{I}$.

Training Phase: $\mathcal{F}_{I}$ performs a series of polynomially bounded number of queries in an adaptive fashion in this phase. The queries allowed are identical to the queries allowed in Phase $\mathbf{1}$ of IND-CLSC-TKEMCCA2-I game.

Forgery: At the end of the Training Phase (which is decided by $\mathcal{F}_{I}$ ), $\mathcal{F}_{I}$ sends to $\mathcal{C}$ an encapsulation $\left\langle\tau^{*}, \psi^{*}, I D_{A^{*}}, I D_{B^{*}}\right\rangle$, where $I D_{A^{*}}$ is the sender identity and $I D_{B^{*}}$ is the receiver identity. It is to be noted that the partial private key of the sender $I D_{A^{*}}$ should not be queried and the public key of $I D_{A^{*}}$ should not be replaced during the Training Phase simultaneously. In addition $\psi^{*}$ should not be the response for any key encapsulation queries by $\mathcal{F}_{I}$ during the Training Phase.

$\mathcal{F}_{I}$ wins the game if the output of $\operatorname{Decap}\left(\psi^{*}, \tau^{*}, I D_{A^{*}}, P K_{A^{*}}, I D_{B^{*}}, P K_{B^{*}}, S_{B^{*}}\right)$ is not invalid. The advantage of $\mathcal{F}_{I}$ is defined as the probability with which it wins the EUF-CLSC-TKEM-CMA-I game.

$\boldsymbol{E} \boldsymbol{U F}$ - CLSC-TKEM-CMA-II: The following is the interactive game between $\mathcal{C}$ and $\mathcal{F}_{I I}$ :

Setup: The challenger $\mathcal{C}$ runs this algorithm to generate the master public and private keys, params and msk respectively. $\mathcal{C}$ gives both params and $m s k$ to $\mathcal{F}_{I I}$.

Training Phase: $\mathcal{F}_{I I}$ performs a series of polynomially bounded number of queries in an adaptive fashion in this phase. The queries allowed are identical to the queries allowed in Phase $\mathbf{1}$ of IND-CLSC-TKEMCCA2-II game.

Forgery: At the end of the Training Phase (which is decided by $\mathcal{F}_{I I}$ ), $\mathcal{F}_{I I}$ sends to $\mathcal{C}$ an encapsulation $\left\langle\tau^{*}, \psi^{*}, I D_{A^{*}}, I D_{B^{*}}\right\rangle$, where $I D_{A^{*}}$ is the sender identity and $I D_{B^{*}}$ is the receiver identity. It is to be noted that $\mathcal{F}_{I I}$ should not query the secret value $x_{A^{*}}$ of the sender $I D_{A^{*}}$ and should not replace the public key of $I D_{A^{*}}$ during the Training Phase. In addition $\psi^{*}$ should not be the response for any key encapsulation queries by $\mathcal{F}_{I I}$ during the Training Phase.

$\mathcal{F}_{I I}$ wins the game if the output of $\operatorname{Decap}\left(\psi^{*}, \tau^{*}, I D_{A^{*}}, P K_{A^{*}}, I D_{B^{*}}, P K_{B^{*}}, S_{B^{*}}\right)$ is not invalid. The advantage of $\mathcal{F}_{I I}$ is defined as the probability with which it wins the EUF-CLSC-TKEM-CMA-II game.

\section{Review and Attack of Lippold et al.'s CLSC-TKEM}

In this section, we review the CL-KEM scheme of Lippold et al., presented in [13]. We show that the scheme in $[13]$ is not CCA secure.

\subsection{Review of the scheme}

This scheme has the following five algorithms.

1. Setup: Given a security parameter $\kappa$, the KGC performs the following to setup the system:

- Chooses two groups $\mathbb{G}_{1}$ and $\mathbb{G}_{2}$ of prime order $p$.

- It also chooses a bilinear map $\hat{e}: \mathbb{G}_{1} \times \mathbb{G}_{1} \rightarrow \mathbb{G}_{2}$.

- Chooses a generator $g \in_{R} \mathbb{G}_{1}$.

- Chooses a suitable Water's hash function $H$, as described in [24].

- Chooses $u_{1}, u_{2}, \alpha \in \mathbb{G}_{1}$, and computes $z=\hat{e}(g, \alpha)$.

- The public parameters of the system are params $=\left\langle\kappa, \mathbb{G}_{1}, \mathbb{G}_{2}, p, g, H, u_{1}, u_{2}, z\right\rangle$ and $\alpha$ is the master secret key. 
2. Identity-Based Key Derivation: Given the master secret key $\alpha$ and the identity $I D \in 0,1^{n}$, the KGC generates an ID-Based private key corresponding to the given identity as follows:

- Chooses $s \in_{R} \mathbb{Z}_{p}^{*}$.

- Returns $s k_{I D}=\left(\alpha H(I D)^{s}, g^{s}\right)$.

3. User Key Generation: This algorithm is executed by the user with identity $I D$, in order to generate his user secret value and the certificateless public key.

- The user chooses a secret value $x_{I D} \in_{R} \mathbb{Z}_{p}^{*}$.

- Computes the certificateless public key $\beta_{I D}=z^{x_{I D}}$.

- Return $\left\langle x_{I D}, \beta_{I D}\right\rangle$.

4. Encapsulation: Given the public key $\beta_{I D}$ of a user with identity $I D$ and a message $M$, the sender generates an encryption key $K$ and the corresponding encapsulation $C$ as follows:

- Chooses $r \in_{R} \mathbb{Z}_{p}^{*}$.

- Computes $c_{1}=g^{r}$ and $c_{2}=H(I D)^{r}$.

- Computes $t=T C R\left(c_{1}\right)$, where $T C R$ is a Target Collision Resistant hash function.

- Computes $c_{3}=\left(u_{1}^{t} u_{2}\right)^{r}$.

- Computes $K=\beta_{I D}^{r}=\left(z^{x}\right)^{r} \in \mathbb{G}_{2}$.

- Computes $C=\left\langle c_{1}, c_{2}, c_{3}\right\rangle \in \mathbb{G}_{1}^{3}$.

- Returns $(K, C)$. (Note that $K$ is the key that is used in a symmetric data encapsulation mechanism (DEM) for the encryption of the message and is not a part of the ciphertext.)

5. Decapsulation: Given the secret keys $d_{1}=\alpha H(I D)^{s}, d_{2}=g^{s}$ and $x_{I D}$, and an encapsulation $C=$ $\left\langle c_{1}, c_{2}, c_{3}\right\rangle$, the receiver of the ciphertext executes this algorithm to recover the key $K$ from $C$ as follows:

- Chooses $r_{1}, r_{2} \in R \mathbb{Z}_{p}^{*}$.

- Computes $t=T C R\left(c_{1}\right)$.

- Computes $K=\left(\frac{\hat{e}\left(c_{1}, d_{1}\left(u_{1}^{t} u_{2}\right)^{r_{1}} H(I D)^{r_{2}}\right)}{\hat{e}\left(c_{2}, d_{2} g^{r_{2}}\right) \hat{e}\left(g^{r_{1}}, c_{3}\right)}\right)^{x_{I D}}$

- Return the key $K$ for data decapsulation.

\subsection{Attack of Lippold et al.'s CL-KEM}

In this section, we show that the CL-KEM by Lippold et al. [13] does not provide confidentiality.

Attack on Confidentiality: During the confidentiality game for Type-I adversary, the adversary is allowed to replace the public key of the receiver. The following attack is possible due to this liberalized constraint on the adversary.

\section{Attack by Type-I adversary:}

- Let $I D^{*}$ be the target identity on which the adversary wishes to be challenged.

- The adversary chooses $x^{\prime} \in R \mathbb{Z}_{p}^{*}$.

- Replaces the public key of $I D^{*}$ as $\beta_{I D^{*}}^{\prime}=\hat{e}(g, g)^{x^{\prime}}$.

- The adversary submits $I D^{*}$ to the challenger as the challenge identity.

Upon receiving the challenge identity $I D^{*}$, the challenger generates the challenge encapsulation $C^{*}$, the encapsulation key $K^{*}$ and sends it to the adversary. On receiving $C^{*}=\left\langle c_{1}^{*}, c_{2}^{*}, c_{3}^{*}\right\rangle$ and $K^{*}$ from the challenger, the Type-I adversary computes the key $K^{\prime}$ from $C^{*}$ as follows and distinguishes $C^{*}$ :

$$
\begin{aligned}
K^{\prime} & =\hat{e}\left(g^{x^{\prime}}, c_{1}\right) \\
& =\hat{e}\left(g^{x^{\prime}}, g^{r}\right) \\
& =\hat{e}(g, g)^{x^{\prime} r} \\
& =\left(\beta_{I D^{*}}^{\prime}\right)^{r}
\end{aligned}
$$

Now, $K^{\prime}$ is the key corresponding to the encapsulation $C^{*}$ generated by the challenger. The adversary compares $K^{\prime}$ with $K^{*}$, and returns $b^{\prime}=1$ if they are identical, $b^{\prime}=0$ otherwise. 


\section{Review and Attack of Fagen Li et al.'s CL-KEM}

In this section we review the CLSC-TKEM scheme of Fagen Li et al, presented in [15]. We also show that the scheme in $[15]$ is existentially forgeable.

\subsection{Review of the scheme}

This scheme has the following seven algorithms.

1. Setup: Given $\kappa$ the security parameter, the KGC chooses two groups $\mathbb{G}_{1}$ and $\mathbb{G}_{2}$ of prime order $q$, a bilinear map $\hat{e}: \mathbb{G}_{1} \times \mathbb{G}_{1} \rightarrow \mathbb{G}_{2}$ and a generator $P \in_{R} \mathbb{G}_{1}$. It then chooses a master private key $s \in \in_{R} \mathbb{Z}_{q}^{*}$, sets a system-wide public key $P_{p u b}=s P$ and chooses four cryptographic hash functions defined by $H_{1}:\{0,1\}^{*} \rightarrow \mathbb{G}_{1}, H_{2}:\{0,1\}^{*} \rightarrow\{0,1\}^{n}, H_{3}:\{0,1\}^{*} \rightarrow \mathbb{G}_{1}$ and $H_{4}:\{0,1\}^{*} \rightarrow \mathbb{G}_{1}$. Here $n$ is the key length of a DEM. The public parameters Params $=\left\langle\mathbb{G}_{1}, \mathbb{G}_{2}, P, \hat{e}, H_{1}, H_{2}, H_{3}, H_{4}, P_{p u b}\right\rangle$.

2. Partial Private Key Extract: Given an identity $I D_{A} \in\{0,1\}^{*}$, the KGC does the following to extract the partial private key corresponding to $I D_{A}$ :

- Computes $Q_{A}=H_{1}\left(I D_{A}\right) \in \mathbb{G}_{1}$.

- Sets the partial private key $D_{A}=s Q_{A}$.

3. Generate User Key: A user with identity $I D_{A}$ chooses $x_{A} \in_{R} \mathcal{Z}_{q}^{*}$ and sets the public key $P K_{A}=x_{A} P$.

4. Set Private Key: The full private key of the user $A$ is set to be $S_{A}=\left(x_{A}, D_{A}\right)$.

5. Sym $\left(I D_{A}, P K_{A}, S_{A}, I D_{B}, P K_{B}\right)$. Given the sender's identity $I D_{A}$, public key $P K_{A}$, private key $S_{A}$, the receiver's identity $I D_{B}$ and public key $P K_{B}$ as input, the algorithm produces the symmetric key $K$ as follows:

- The sender $A$ chooses $r \in_{R} \mathcal{Z}_{q}^{*}$,

- Computes $U=r P$ and $T=\hat{e}\left(P_{p u b}, Q_{B}\right)^{r}$,

- Computes $K=H_{2}\left(U, T, r\left(P K_{B}\right), I D_{B}, P K_{B}\right)$,

- Outputs $K$ and a set $\omega=\left\langle r, U, I D_{A}, P K_{A}, S_{A}, I D_{B}, P K_{B}\right\rangle$

6. Encap $(\omega, \tau)$. Given a state information $\omega$ and an arbitrary $\operatorname{tag} \tau$, the sender $A$ obtains the encapsulation $\psi$ by performing the following:

- Computes $H=H_{3}\left(U, \tau, I D_{A}, P K_{A}\right)$.

- Computes $H^{\prime}=H_{4}\left(U, \tau, I D_{A}, P K_{A}\right)$.

- Computes $W=D_{A}+r H+x_{A} H^{\prime}$.

- Output $\psi=\langle U, W\rangle$

7. Decap $\left(\psi, \tau, I D_{A}, P K_{A}, I D_{B}, P K_{B}, S_{B}\right)$. Given the encapsulation $\psi$, a tag $\tau$, the sender's identity $I D_{A}$, public key $P K_{A}$, the receiver's identity $I D_{B}$, public key $P K_{B}$ and private key $S_{B}$ the key $K$ is computed by the receiver $B$ as follows:

- Computes $H=H_{3}\left(U, \tau, I D_{A}, P K_{A}\right)$.

- Computes $H^{\prime}=H_{4}\left(U, \tau, I D_{A}, P K_{A}\right)$.

- If $\hat{e}\left(P_{p u b}, Q_{A}\right) \hat{e}(U, H) \hat{e}\left(P K_{A}, H^{\prime}\right) \stackrel{?}{=} \hat{e}(P, W)$, computes the value $T=\hat{e}\left(D_{B}, U\right)$ and outputs $K=$ $H_{2}\left(U, T, x_{B} U, I D_{B}, P K_{B}\right)$, otherwise outputs invalid.

\subsection{Attack of Fagen Li et al.'s CLSC-TKEM}

We show that the CLSC-TKEM by Fagen Li et al. [15] is existentially forgeable in this section.

Attack on Unforgeability: Fagen Li et al. [15] have claimed that their scheme is existentially unforgeable against both Type-I and Type-II attacks. We show that the scheme does not resist both Type-I and Type-II attacks. It is to be noted that, in the unforgeability games, EUF-CLSC-TKEM-CMA-I and EUF-CLSCTKEM-CMA-II the corresponding forgers $\mathcal{F}_{I}$ and $\mathcal{F}_{I I}$ have access to the full private key of the receiver $B$, also, $\mathcal{F}_{I}$ is not allowed to extract the partial private keys of the sender $A$ and $\mathcal{F}_{I I}$ is not allowed to extract the user secret key or replace the sender $A$ 's public key. These constraints are maintained in order to ensure insider security.

Attack by Type-I forger $\mathcal{F}_{I}$ : During the EUF-CLSC-TKEM-CMA-I game, the forger $\mathcal{F}_{I}$ interacts with the challenger $\mathcal{C}$ during the Training Phase. $\mathcal{F}_{I}$ has access to the various oracles offered by $\mathcal{C}$ in addition to it, $\mathcal{F}_{I}$ has access to the full private key of the receiver too. 
- During the Training Phase $\mathcal{F}_{I}$ queries $\mathcal{C}$ for an encapsulation with $I D_{A}$ as sender and $I D_{B}$ as receiver with an arbitrary $\operatorname{tag} \tau$.

- Here, the private key of $I D_{A}$ is not queried by $\mathcal{F}_{I}$ and the corresponding public key is not replaced.

$-\mathcal{C}$ responds with $\psi=\langle U, W\rangle$.

Now, $\mathcal{F}_{I}$ obtains a forged encapsulation from the encapsulation $\psi$ received during the Training Phase for the same tag $\tau$, by performing the following steps:

- Let $I D_{B^{*}}$ be a user whose full private key $S_{B^{*}}$ is known to $\mathcal{F}_{I}$.

- $\mathcal{F}_{I}$ computes a new key $K^{\prime}=H_{2}\left(U, T^{\prime}, x_{B^{*}} U, I D_{B^{*}}, P K_{B^{*}}\right)$, where $T^{\prime}=\hat{e}\left(D_{B^{*}}, U\right)$.

- Now, $\psi^{*}=\langle U, W\rangle$ is a valid encapsulation of the key $K^{\prime}$ from the sender $I D_{A}$ to a new receiver $I D_{B^{*}}$.

The correctness of the attack can be easily verified because Decap $\left(\psi^{*}, \tau, I D_{A}, P K_{A}, I D_{B^{*}}, P K_{B^{*}}, S_{B^{*}}\right)$ passes the verification and yields a different key $K^{\prime}$ as follows.

- Compute $H=H_{3}\left(U, \tau, I D_{A}, P K_{A}\right)$ and $H^{\prime}=H_{4}\left(U, \tau, I D_{A}, P K_{A}\right)$. It is to be noted that the computation of $H$ and $H^{\prime}$ will yield the same value for both ciphertexts $\psi$ and $\psi^{*}$ because both the computations do not use the receiver identity and public key. Also, the value of $U$ is same in both the ciphertexts.

- The validity check $\hat{e}\left(P_{p u b}, Q_{A}\right) \hat{e}(U, H) \hat{e}\left(P K_{A}, H^{\prime}\right) \stackrel{?}{=} \hat{e}(P, W)$ also holds because this verification is also dependent on the sender's identity and public key alone and no receiver component is used explicitly or implicitly.

The value $T^{*}=\hat{e}\left(D_{B^{*}}, U\right)$ is computed and $K^{\prime}=H_{2}\left(U, T^{\prime}, x_{B^{*}} U, I D_{B^{*}}, P K_{B^{*}}\right)$ is output as the key. Thus $\psi^{*}$ is a valid forgery with respect to the new key $K^{\prime}$.

Attack by Type-II forger $\mathcal{F}_{I I}$ : The attack by Type-II forger is identical to that of the attack by the Type-I forger $\mathcal{F}_{I}$ because as mentioned above a Type-II forger $\mathcal{F}_{I I}$ also has access to the full private key of the receiver $B$. The forgery can be done in a similar way as described in Attack by Type-I forger $\mathcal{F}_{I}$ because the attack does not involve the user secret value of the sender $A$, which is not available to the forger $\mathcal{F}_{I I}$. Remark: We also point out that the same weakness holds for the generic Certificateless Hybrid Signcryption scheme proposed in [15]. The weakness is due to the lack of binding of the receiver identity to the signature component of the encapsulation $\psi$.

\section{Improved CLSC-TKEM Scheme (ICLSC-TKEM)}

In the preceding section we saw that the CLSC-TKEM scheme proposed by Fagen Li et al. does not withstand chosen message attack. The weakness of the scheme was due to the lack of binding between the receiver identity and the signature generated by the sender. This is the reason, for an encapsulation $\psi$ to act as a valid encapsulation for different keys $K_{i}$ (for $i=1$ to $n$, where $n$ is the number of forged keys) from a single sender to $n$ different receivers. This weakness can be eliminated by making the following changes in the $\boldsymbol{S y m}$, Encap and Decap algorithms in Fagen Li et al's [15] scheme.

Sym $\left(I D_{A}, P K_{A}, S_{A}, I D_{B}, P K_{B}\right)$. Given the sender's identity $I D_{A}$, public key $P K_{A}$, private key $S_{A}$, the receiver's identity $I D_{B}$ and public key $P K_{B}$ as input, the algorithm produces the symmetric key $K$ as follows:

- The sender $A$ chooses $r \in_{R} \mathcal{Z}_{q}^{*}$,

- Computes $U=r P$ and $T=\hat{e}\left(P_{p u b}, Q_{B}\right)^{r}$,

- Computes $K=H_{2}\left(U, T, r\left(P K_{B}\right), I D_{B}, P K_{B}\right)$,

- Outputs $K$ and a set $\omega=\left(r, U, T, I D_{A}, P K_{A}, S_{A}, I D_{B}, P K_{B}\right)$

Encap $(\omega, \tau)$. Given a state information $\omega$ and an arbitrary tag $\tau$, the sender $A$ obtains the encapsulation $\psi$ by performing the following:

- Computes $H=H_{3}\left(U, \tau, T, I D_{A}, P K_{A}, I D_{B}, P K_{B}\right)$.

- Computes $H^{\prime}=H_{4}\left(U, \tau, T, I D_{A}, P K_{A}, I D_{B}, P K_{B}\right)$.

- Computes $W=D_{A}+r H+x_{A} H^{\prime}$.

- Output $\psi=\langle U, W\rangle$ 
Now, it is not possible for $\mathcal{F}_{I}$ and $\mathcal{F}_{I I}$ to generate different forged keys from a sender $I D_{A}$, whose secret key is not known to any receivers as the identity $I D_{B}$ and the public key $P K_{B}$ of the receiver is bound to the signature part of the encapsulation $\psi$ which cannot be altered.

Decap $\left(\psi, \tau, I D_{A}, P K_{A}, I D_{B}, P K_{B}, S_{B}\right)$. Given the encapsulation $\psi$, a tag $\tau$, the sender's identity $I D_{A}$, public key $P K_{A}$, the receiver's identity $I D_{B}$, public key $P K_{B}$ and private key $S_{B}$ the key $K$ is computed as follows:

- Computes the value $T=\hat{e}\left(D_{B}, U\right)$.

- Computes $H=H_{3}\left(U, \tau, T, I D_{A}, P K_{A}, I D_{B}, P K_{B}\right)$.

- Computes $H^{\prime}=H_{4}\left(U, \tau, T, I D_{A}, P K_{A}, I D_{B}, P K_{B}\right)$.

- If $\hat{e}\left(P_{p u b}, Q_{A}\right) \hat{e}(U, H) \hat{e}\left(P K_{A}, H^{\prime}\right) \stackrel{?}{=} \hat{e}(P, W)$ and outputs $K=H_{2}\left(U, T, x_{B} U, I D_{B}, P K_{B}\right)$, otherwise outputs invalid.

\section{Security of the Improved CLSC-TKEM Scheme}

In this section we provide the formal proof for the confidentiality and unforgeability of the improved CLSCTKEM.

\subsection{Type-I Confidentiality}

Theorem 1. If an IND-ICLSC-TKEM-CCA2-I adversary $\mathcal{A}_{I}$ has an advantage $\epsilon$ against the IND-ICLSCTKEM-CCA2-I security of the ICLSC-TKEM scheme, asking $q_{H_{i}}(i=1,2,3,4)$ hash queries to random oracles $\mathcal{O}_{H_{i}}(i=1,2,3,4), q_{p p k}$ partial private key extract queries and $q_{f p k}$ private key extract queries, then there exist an algorithm $\mathcal{C}$ that solves the $C B D H$ problem with the following advantage

$$
\epsilon^{\prime} \geq \epsilon\left(1-\frac{q_{p p k}}{q_{H_{1}}}\right)\left(1-\frac{q_{f p k}}{q_{H_{1}}}\right)\left(\frac{1}{q_{H_{1}}-\left(q_{p p k}+q_{f p k}\right)}\right)\left(\frac{1}{q_{H_{2}}}\right)
$$

Proof: A challenger $\mathcal{C}$ is challenged with an instance of the $\mathrm{CBDH}$ problem. Given $\langle P, a P, b P, c P\rangle \in \mathbb{G}_{1}$, $\mathcal{C}$ has to find out $\hat{e}(P, P)^{a b c}$. Let $\mathcal{A}_{I}$ be the adversary who is capable of breaking the IND-ICLSC-TKEMCCA2-I security of the ICLSC-TKEM scheme. $\mathcal{C}$ can make use of $\mathcal{A}_{I}$ to find the solution of the CBDH problem instance by playing the following interactive game with $\mathcal{A}_{I}$.

Setup: $\mathcal{C}$ sets the master public key $P_{\text {pub }}=a P$, designs the hash functions $H_{i}(i=1$ to 4$)$ as random oracles $\mathcal{O}_{H_{i}}(i=1$ to 4$)$ respectively. In order to maintain the consistency between the responses to the hash queries, $\mathcal{C}$ maintains lists $L_{i}(i=1$ to 4$)$ and to maintain the list of issued private keys and public keys, $\mathcal{C}$ maintains a list $L_{K} \cdot \mathcal{C}$ gives the public parameters params to $\mathcal{A}_{I}$.

Phase 1: $\mathcal{A}_{I}$ performs a series of polynomially bounded number of queries in an adaptive fashion in this phase. The oracles and queries allowed are described below.

- $\mathcal{O}_{H_{1}}\left(I D_{i}\right)$ : We will make a simplifying assumption that $\mathcal{A}_{I}$ queries the $\mathcal{O}_{H_{1}}$ oracle with distinct identities in each query. Without loss of generality, if the oracle query is repeated with an already queried identity, by definition the oracle consults the list $L_{1}$ and gives the same response. Thus, we assume that $\mathcal{A}_{I}$ asks $q_{H_{1}}$ distinct queries for $q_{H_{1}}$ distinct identities. Among this $q_{H_{1}}$ identities, a random identity has to be selected by $\mathcal{C}$ as target identity and it is done as follows (Note that $\mathcal{A}_{I}$ should also choose this identity in the challenge phase).

$\mathcal{C}$ selects a random index $\gamma$, where $1 \leq \gamma \leq q_{H_{1}}$. $\mathcal{C}$ does not reveal $\gamma$ to $\mathcal{A}_{I}$. When $\mathcal{A}_{I}$ puts forth the $\gamma^{\text {th }}$ query on $I D_{\gamma}, \mathcal{C}$ decides to fix $I D_{\gamma}$ as target identity for the challenge phase. Moreover, $\mathcal{C}$ responds to $\mathcal{A}_{I}$ as follows:

- If it is the $\gamma^{\text {th }}$ query, then $\mathcal{C}$ sets $Q_{\gamma}=b P$ and stores the tuple $\left\langle I D_{\gamma}, Q_{\gamma}=b P,-\right\rangle$ in the list $L_{1}$. Here, $\mathcal{C}$ does not know $b . \mathcal{C}$ is simply using the $b P$ value given in the instance of the CBDH problem.

- For all other queries, $\mathcal{C}$ chooses $b_{i} \in_{R} Z_{q}^{*}$ and sets $Q_{i}=b_{i} P$ and stores $\left\langle I D_{i}, Q_{i}, b_{i}\right\rangle$ in the list $L_{1}$. 
$\mathcal{C}$ returns $Q_{i}$ to $\mathcal{A}$. (Note that as the identities are assumed to be distinct, for each query, we create distinct entry and add in the list $\left.L_{1}\right)$.

- $\mathcal{O}_{H_{2}}\left(U, T, r\left(P K_{B}\right), I D_{B}, P K_{B}\right)$ : To respond to this query, $\mathcal{C}$ checks whether a tuple of the form $\left\langle U, T, r\left(P K_{B}\right), I D_{B}, P K_{B}, K\right\rangle$ exists in list $L_{2}$. If so, returns $K$ to $\mathcal{A}_{I}$ else chooses $K \in_{R}\{0,1\}^{n}$, adds the tuple $\left\langle U, T, r\left(P K_{B}\right), I D_{B}, P K_{B}, K\right\rangle$ to the list $L_{2}$ and returns $K$ to $\mathcal{A}_{I}$.

- $\mathcal{O}_{H_{3}}\left(U, \tau, T, I D_{A}, P K_{A}, I D_{B}, P K_{B}\right)$ : To respond to this query, $\mathcal{C}$ checks whether a tuple of the form $\left\langle U, \tau, T, I D_{A}, P K_{A}, I D_{B}, P K_{B}, h_{i}, H\right\rangle$ exists in the list $L_{3}$. If so, returns $H$ to $\mathcal{A}_{I}$ else performs the following:

- If $I D_{B} \neq I D_{\gamma}, \mathcal{C}$ chooses $h_{i} \in_{R} \mathbb{Z}_{q}^{*}$, adds the tuple $\left\langle U, \tau, T, I D_{A}, P K_{A}, I D_{B}, P K_{B}, h_{i}, H=h_{i} P\right\rangle$ to the list $L_{3}$ and returns $H$ to $\mathcal{A}_{I}$.

- If $I D_{B}=I D_{\gamma}, \mathcal{C}$ chooses $h_{i} \in_{R} \mathbb{Z}_{q}^{*}$, adds the tuple $\left\langle U, \tau, T, I D_{A}, P K_{A}, I D_{B}, P K_{B}, h_{i}, H=\right.$ $\left.h_{i} P_{\text {pub }}\right\rangle$ to the list $L_{3}$ and returns $H$ to $\mathcal{A}_{I}$.

- $\mathcal{O}_{H_{4}}\left(U, \tau, T, I D_{A}, P K_{A}, I D_{B}, P K_{B}\right)$ : To respond to this query, $\mathcal{C}$ checks whether a tuple of the form $\left\langle U, \tau, T, I D_{A}, P K_{A}, I D_{B}, P K_{B}, h_{i}^{\prime}, H^{\prime}\right\rangle$ exists in the list $L_{4}$. If so, returns $H^{\prime}$ to $\mathcal{A}_{I}$ else chooses $h_{i}^{\prime} \in_{R} \mathbb{Z}_{q}^{*}$, adds the tuple $\left\langle U, \tau, T, I D_{A}, P K_{A}, I D_{B}, P K_{B}, h_{i}^{\prime}, H^{\prime}=h_{i}^{\prime} P\right\rangle$ to the list $L_{4}$ and returns $H^{\prime}$ to $\mathcal{A}_{I}$.

- $\mathcal{O}_{\text {ExtractPartialPrivateKey }}\left(I D_{i}\right)$ : On a request for the partial private key of a user with identity $I D_{i}$, $\mathcal{C}$ aborts if $I D_{i}=I D_{\gamma}$. Else, $\mathcal{C}$ retrieves the tuple $\left\langle I D_{i}, Q_{i}, b_{i}\right\rangle$ from list $L_{1}$, returns $D_{i}=b_{i} a P=a Q_{i}$ and adds the tuple $\left\langle I D_{i},-, D_{i},-\right\rangle$ to the list $L_{K}$.

(Note: It is assumed throughout the confidentiality game, $\mathcal{A}_{I}$ queries $\mathcal{O}_{H_{1}}$ oracle with $I D_{i}$ before querying any other oracles with $I D_{i}$ as input.)

- $\mathcal{O}_{\text {RequestPublicKey }}\left(I D_{i}\right): \mathcal{A}_{I}$ produces an identity $I D_{i}$ to $\mathcal{C}$ and requests $I D_{i}$ 's public key. $\mathcal{C}$ checks in the list $L_{K}$ for an tuple of the form $\left\langle I D_{i}, x_{i}, D_{i}, P K_{i}\right\rangle$. If an entry exists, then responds by returning the corresponding public key $P K_{i}$ to $\mathcal{A}_{I}$. If it does not exist, $\mathcal{C}$ chooses $x_{i} \in_{R} \mathbb{Z}_{q}^{*}$, sets $P K_{i}=x_{i} P$, adds the tuple $\left\langle I D_{i}, x_{i},-, P K_{i}\right\rangle$ (note that $D_{i}$ is computed only after the $\mathcal{O}_{\text {ExtractPartialPrivateKey }}\left(I D_{i}\right)$ query) to the list $L_{K}$ and sends the corresponding $P K_{i}$ to $\mathcal{A}_{I}$.

- $\mathcal{O}_{\text {ExtractPrivateKey }}\left(I D_{i}\right): \mathcal{A}_{I}$ produces an identity $I D_{i}$ and requests the corresponding full private key. If $I D_{i}$ 's public key has not been replaced and if $I D_{i} \neq I D_{\gamma}$, then $\mathcal{C}$ responds with the full private key $S_{i}=\left\langle x_{i}, D_{i}\right\rangle$ retrieving it from the list $L_{K}$. If $\mathcal{A}_{I}$ has already replaced $I D_{i}$ 's public key, then $\mathcal{C}$ does not provide the corresponding private key to $\mathcal{A}_{I}$ (Note that $\mathcal{A}_{I}$ is allowed to replace the public key of any identity including $I D_{\gamma}$, thus $\mathcal{A}_{I}$ knows the user secret value corresponding to any identity, including $\left.I D_{\gamma}\right)$. If $I D_{i}=I D_{\gamma}$ then $\mathcal{C}$ aborts.

- $\mathcal{O}_{\text {ReplacePublicKey }}\left(P K_{i}^{\prime}\right)$ : In order to replace the public key $P K_{i}$ of a user $I D_{i}$ with any value $P K_{i}^{\prime}$ of $\mathcal{A}_{I}$ 's choice, $\mathcal{C}$ updates the corresponding tuple in the list $L_{K}$ as $\left\langle I D_{i},-, D_{i}, P K_{i}^{\prime}\right\rangle$. The current value of the user's public key is used by $\mathcal{C}$ in for computations or responses to any queries made by $\mathcal{A}_{I}$.

- $\mathcal{O}_{\text {SymmetricKeyGeneration }}: \mathcal{A}_{I}$ produces a sender's identity $I D_{A}$, public key $P K_{A}$, the receiver's identity $I D_{B}$ and public key $P K_{B}$ to $\mathcal{C}$. Now, $\mathcal{C}$ computes the symmetric key $K$ and an internal state information $\omega$, stores and keeps $\omega$ secret from the view of $\mathcal{A}_{I}$ and sends the symmetric key $K$ to $\mathcal{A}_{I}$. It is to be noted that $\mathcal{C}$ can perform this even if $\mathcal{C}$ does not know the private key corresponding to the sender $I D_{A}$ or the receiver $I D_{B}$ because computing $K$ does not involve the private key of either the sender or receiver.

- $\mathcal{O}_{\text {KeyEncapsulation }}: \mathcal{A}_{I}$ produces an arbitrary tag $\tau$, the sender's identity $I D_{A}$, public key $P K_{A}$, the receiver's identity $I D_{B}$ and public key $P K_{B}$ to $\mathcal{C}$. The full private key of the sender $S_{A}=\left(x_{A}, D_{A}\right)$ is obtained from the list $L_{K} \cdot \mathcal{C}$ checks whether a corresponding $\omega$ value is stored previously.

- If $\omega$ does not exist, $\mathcal{C}$ returns invalid.

- If a corresponding $\omega$ exists and $I D_{A} \neq I D_{\gamma}$, then $\mathcal{C}$ computes the encapsulation $\psi$ with $\omega$ and $\tau$ as per the actual encapsulation algorithm, and deletes $\omega$.

- If a corresponding $\omega$ exists and $I D_{A}=I D_{\gamma}$, then $\mathcal{C}$ performs the following to compute $\psi$ (It is to be noted that $\mathcal{C}$ does not know the private key corresponding to $I D_{\gamma}$, so it cooks up the encapsulation in a different way): 
* Chooses $r, h_{i}, h_{i}^{\prime} \in_{R} \mathcal{Z}_{q}^{*}$ and computes $U=r P-h_{i}^{-1} Q_{A}$, where $Q_{A}=b P$ is obtained from the list $L_{1}$.

* Computes $H=h_{i} P_{p u b}$ and adds the tuple $\left\langle U, \tau, T, I D_{A}, P K_{A}, I D_{B}, P K_{B}, h_{i}, H\right\rangle$ to the list $L_{3}$.

* Computes $H^{\prime}=h_{i}^{\prime} P$ and adds the tuple $\left\langle U, \tau, T, I D_{A}, P K_{A}, I D_{B}, P K_{B}, h_{i}^{\prime}, H^{\prime}\right\rangle$ to the list $L_{4}$.

* Computes $W=r H+h_{i}^{\prime} P K_{A}$ (This is possible because $\mathcal{C}$ knows the public key $P K_{A}$ of the sender $A$ which is $\left.x_{A} P\right)$.

* Outputs, $\psi=\langle U, W\rangle$ as the encapsulation.

We show that, $\psi=\langle U, W\rangle$ passes the verification done by $\mathcal{A}_{I}$ to validate the encapsulation, because the equality $\hat{e}\left(P_{\text {pub }}, Q_{A}\right) \hat{e}(U, H) \hat{e}\left(P K_{A}, H^{\prime}\right) \stackrel{?}{=} \hat{e}(P, W)$ holds.

\section{Correctness:}

$$
\begin{aligned}
\hat{e}\left(P_{p u b}, Q_{A}\right) \hat{e}(U, H) \hat{e}\left(P K_{A}, H^{\prime}\right) & =\hat{e}(a P, b P) \hat{e}\left(r P-h_{i}^{-1} Q_{A}, H\right) \hat{e}\left(x_{A} P, H^{\prime}\right) \\
& =\hat{e}(a P, b P) \hat{e}(r P, H) \hat{e}\left(-h_{i}^{-1} Q_{A}, H\right) \hat{e}\left(x_{A} P, H^{\prime}\right) \\
& =\hat{e}(a P, b P) \hat{e}\left(r P, h_{i} a P\right) \hat{e}\left(h_{i}^{-1} b P, h_{i} a P\right)^{-1} \hat{e}\left(x_{A} P, h_{i}^{\prime} P\right) \\
& =\hat{e}(a P, b P) \hat{e}\left(r P, h_{i} a P\right) \hat{e}(a P, b P)^{-1} \hat{e}\left(x_{A} P, h_{i}^{\prime} P\right) \\
& =\hat{e}\left(r P, h_{i} a P\right) \hat{e}\left(x_{A} P, h_{i}^{\prime} P\right) \\
& =\hat{e}\left(P, h_{i} a r P+x_{A} h_{i}^{\prime} P\right) \\
& =\hat{e}\left(P, r H+h_{i}^{\prime} P K_{A}\right) \\
& =\hat{e}(P, W)
\end{aligned}
$$

- $\mathcal{O}_{\text {KeyDecapsulation }}: \mathcal{A}_{I}$ produces an encapsulation $\psi$, a tag $\tau$, the sender's identity $I D_{A}$, public key $P K_{A}$, the receiver's identity $I D_{B}$ and public key $P K_{B}$ to $\mathcal{C}$. The private key of the receiver $S_{B}$ is obtained from the list $L_{K}$. It is to be noted that $\mathcal{C}$ may not be aware of the corresponding private key if the public key of $I D_{B}$ is replaced. In this case $\mathcal{C}$ obtains the private key of $I D_{B}$ from $\mathcal{A}_{I}$.

- If $I D_{B} \neq I D_{\gamma}$, then $\mathcal{C}$ computes the decapsulation of $\psi$ as per the actual decapsulation algorithm.

- If $I D_{B}=I D_{\gamma}$, then $\mathcal{C}$ computes $K$ from $\psi$ as follows:

* Searches in the list $L_{3}$ and $L_{4}$ for entries of the type $\left\langle U, \tau, T, I D_{A}, P K_{A}, I D_{B}, P K_{B}, h_{i}, H\right\rangle$ and $\left\langle U, T, \tau, I D_{A}, P K_{A}, I D_{B}, P K_{B}, h_{i}^{\prime}, H^{\prime}\right\rangle$ respectively.

* If entries $H$ and $H^{\prime}$ exist then $\mathcal{C}$ checks whether the equality $\hat{e}\left(P_{p u b}, Q_{A}\right) \hat{e}(U, H) \hat{e}\left(P K_{A}, H^{\prime}\right) \stackrel{?}{=}$ $\hat{e}(P, W)$ holds.

* If the above equality holds, then retrieves the corresponding value of $T$ from the lists $L_{3}$ and $L_{4}$ (note that both the $T$ values should be equal).

* Now, $\mathcal{C}$ checks whether a tuple of the form $\left\langle U, T, x_{B} U, I D_{B}, P K_{B}, K\right\rangle$ exists in the list $L_{2}$. If it exists output the corresponding $K$ value as the decapsulation of $\psi$.

Challenge: At the end of Phase $1, \mathcal{A}_{I}$ sends to $\mathcal{C}$, a sender identity $I D_{A^{*}}$ and a receiver identity $I D_{B^{*}}$ on which $\mathcal{A}_{I}$ wishes to be challenged. Here, the full private key of the receiver $I D_{B^{*}}$ was not queried in Phase 1. $\mathcal{C}$ aborts the game if $I D_{B^{*}} \neq I D_{\gamma}, \mathcal{C}$ performs the following to compute the challenge encapsulation $\psi^{*}$.

- Sets $U=c P$ and chooses $T \in_{R} \mathbb{G}_{2}$.

- Chooses $K_{0} \in_{R} \mathcal{K}$, where $\mathcal{K}$ is the key space of the ICLSC-TKEM scheme

- Computes $K_{1}=H_{2}\left(U, T, x_{B} U, I D_{B}, P K_{B}\right)$.

- Sets $\omega^{*}=\left\langle-, U, U^{\prime}, T, I D_{A}, P K_{A}, S_{A}, I D_{B}, P K_{B}\right\rangle$.

- Now, $\mathcal{C}$ chooses a bit $\delta \in_{R}\{0,1\}$ and sends $K_{\delta}$ to $\mathcal{A}_{I}$.

- $\mathcal{A}_{I}$ generates an arbitrary tag $\tau^{*}$ and sends it to $\mathcal{C}$.

- Chooses $h_{i}, h_{i}^{\prime} \in_{R} \mathbb{Z}_{q}^{*}$, stores the tuple $\left\langle U, \tau^{*}, T, I D_{A}, P K_{A}, I D_{B}, P K_{B}, h_{i}, H=h_{i} P\right\rangle$ to the list $L_{3}$ and $\left\langle U, \tau^{*}, T, I D_{A}, P K_{A}, I D_{B}, P K_{B}, h_{i}^{\prime}, H^{\prime}=h_{i}^{\prime} P\right\rangle$ to the list $L_{4}$.

- Since $\mathcal{C}$ knows the private key of the sender, $\mathcal{C}$ computes $W=D_{A}+h_{i} c P+h_{i}^{\prime} x_{A} P$.

- $\mathcal{C}$ now sends $\psi^{*}=\langle U, W\rangle$ to $\mathcal{A}_{I}$.

Phase II: $\mathcal{A}_{I}$ adaptively queries the oracles as in Phase I, consistent with the constraints for Type-I adversary. Besides this it cannot query decapsulation on $\psi^{*}$.

Guess: Since $\mathcal{A}_{I}$ is capable of breaking the IND-ICLSC-TKEM-CCA2-I security of ICLSC-TKEM (which is assumed at the beginning of the proof), $\mathcal{A}_{I}$ should have queried $\mathcal{O}_{H_{2}}$ with $\left(U, T, x_{B} U, I D_{B}, P K_{B}\right)$ as inputs. it is to be noted that $T=\hat{e}\left(P_{p u b}, Q_{B}\right)^{r}=\hat{e}(a P, b P)^{c}$. Therefore, if the list $L_{2}$ has $q_{H_{2}}$ queries 
corresponding to the sender $I D_{A}$ and receiver $I D_{B}$, one of the $T$ 's among $q_{H_{2}}$ values stored in the list $L_{2}$, is the solution for the $\mathrm{CBDH}$ problem instance. Now, $\mathcal{C}$ chooses one $T$ value uniformly at random from the $q_{H_{2}}$ values from the list $L_{2}$ and outputs it as the solution for the CBDH instance.

Analysis: We now assess the probability of success of the challenger $\mathcal{C}$. The events in which $\mathcal{C}$ aborts the IND-ICLSC-TKEM-CCA2-I game are,

1. $E_{1}$ - when $\mathcal{A}_{I}$ queries the partial private key of the target identity $I D_{\gamma}$ and $\operatorname{Pr}\left[E_{1}\right]=\frac{q_{p p k}}{q_{H_{1}}}$.

2. $E_{2}$ - when $\mathcal{A}_{I}$ queries the full private key of the target identity $I D_{\gamma}$ and $\operatorname{Pr}\left[E_{2}\right]=\frac{q_{f p k}}{q_{H_{1}}}$.

3. $E_{3}$ - when $\mathcal{A}_{I}$ does not choose the target identity $I D_{\gamma}$ as the receiver during the challenge and $\operatorname{Pr}\left[E_{3}\right]=$ $\left(1-\frac{1}{q_{H_{1}}-\left(q_{p p k}+q_{f p k}\right)}\right)$.

The probability that, $\mathcal{C}$ does not abort the IND-ICLSC-TKEM-CCA2-I game is given by

$$
\left(\operatorname{Pr}\left[\neg E_{1} \wedge \neg E_{2} \wedge \neg E_{3}\right]\right)=\left(1-\frac{q_{p p k}}{q_{H_{1}}}\right)\left(1-\frac{q_{f p k}}{q_{H_{1}}}\right)\left(\frac{1}{q_{H_{1}}-\left(q_{p p k}+q_{f p k}\right)}\right)
$$

The probability that, the $T$ chosen randomly from $L_{2}$ by $\mathcal{C}$, being the solution to CBDHP is $\left(\frac{1}{q_{H_{2}}}\right)$. Therefore, the probability of $\mathcal{C}$ solving CBDHP is given by,

$$
\operatorname{Pr}\left[\mathcal{C}(P, a P, b P, c P)=\hat{e}(P, P)^{a b c}\right]=\epsilon\left(1-\frac{q_{p p k}}{q_{H_{1}}}\right)\left(1-\frac{q_{f p k}}{q_{H_{1}}}\right)\left(\frac{1}{q_{H_{1}}-\left(q_{p p k}+q_{f p k}\right)}\right)\left(\frac{1}{q_{H_{2}}}\right)
$$

Since $\epsilon$ is non-negligible, the probability of $\mathcal{C}$ solving CBDHP is also non-negligible.

\subsection{Type-II Confidentiality}

Theorem 2. If an IND-ICLSC-TKEM-CCA2-II adversary $\mathcal{A}_{I I}$ has an advantage $\epsilon$ against the IND-ICLSCTKEM-CCA2-II security of the ICLSC-TKEM scheme, asking $q_{H_{i}}(i=1,2,3,4)$ hash queries to random oracles $\mathcal{O}_{H_{i}}(i=1,2,3,4), q_{f p k}$ full private key extract queries and $q_{r p k}$ replace public key queries, then there exist an algorithm $\mathcal{C}$ that solves the $C D H$ problem with the following advantage

$$
\epsilon^{\prime} \geq \epsilon\left(1-\frac{q_{f p k}}{q_{H_{1}}}\right)\left(1-\frac{q_{r p k}}{q_{H_{1}}}\right)\left(\frac{1}{q_{H_{1}}-\left(q_{f p k}+q_{r p k}\right)}\right)\left(\frac{1}{q_{H_{2}}}\right)
$$

Proof: A challenger $\mathcal{C}$ is challenged with an instance of the $\mathrm{CDH}$ problem say, given $\langle P, a P, b P\rangle \in \mathbb{G}_{1}, \mathcal{C}$ has to find out $P^{a b}$. Let $\mathcal{A}_{I I}$ be the adversary who is capable of breaking the IND-ICLSC-TKEM-CCA2-II security of the ICLSC-TKEM scheme. $\mathcal{C}$ can make use of $\mathcal{A}_{I I}$ to solve the $\mathrm{CDH}$ problem instance by playing the following interactive game, with $\mathcal{A}_{I I}$.

Setup: $\mathcal{C}$ chooses $s \in_{R} \mathbb{Z}_{q}^{*}$, sets the master public key $P_{\text {pub }}=s P$ and gives $s$ to $\mathcal{A}_{I I}$. The hash functions $H_{i}(i=1$ to 4$)$ are viewed as random oracles $\mathcal{O}_{H_{i}}(i=1$ to 4$)$ respectively. In order to maintain the consistency between the responses to the hash queries, $\mathcal{C}$ maintains lists $L_{i}(i=1$ to 4$)$ and to maintain the list of issued private keys and public keys, $\mathcal{C}$ maintains a list $L_{K} \cdot \mathcal{C}$ gives the public parameters params to $\mathcal{A}_{I I}$.

Phase 1: $\mathcal{A}_{I I}$ performs a series of polynomially bounded number of queries in an adaptive fashion in this phase. The oracles and queries allowed are described below.

- $\mathcal{O}_{H_{1}}\left(I D_{i}\right)$ : When $\mathcal{A}_{I I}$ queries the $\mathcal{O}_{H_{1}}$ oracle, $\mathcal{C}$ chooses $b_{i} \in_{R} Z_{q}^{*}$ and sets $Q_{i}=b_{i} P$ and stores $\left\langle I D_{i}, Q_{i}, b_{i}\right\rangle$ in the list $L_{1}$. $\mathcal{C}$ returns $Q_{i}$ to $\mathcal{A}$.

- $\mathcal{O}_{H_{2}}\left(U, T, r\left(P K_{B}\right), I D_{B}, P K_{B}\right):$ To respond to this query, $\mathcal{C}$ checks whether a tuple of the form $\langle U$, $\left.T, r\left(P K_{B}\right), I D_{B}, P K_{B}, K\right\rangle$ exists in list $L_{2}$. If so, returns $K$ to $\mathcal{A}_{I}$ else chooses $K \in_{R}\{0,1\}^{n}$, adds the tuple $\left\langle U, T, r\left(P K_{B}\right), I D_{B}, P K_{B}, K\right\rangle$ to the list $L_{2}$ and returns $K$ to $\mathcal{A}_{I I}$. 
- $\mathcal{O}_{H_{3}}\left(U, \tau, T, I D_{A}, P K_{A}, I D_{B}, P K_{B}\right)$ : To respond to this query, $\mathcal{C}$ checks whether a tuple of the form $\left\langle U, \tau, T, I D_{A}, P K_{A}, I D_{B}, P K_{B}, h_{i}, H\right\rangle$ exists in the list $L_{3}$. If so, returns $H$ to $\mathcal{A}_{I}$ else chooses $h_{i} \in_{R} \mathbb{Z}_{q}^{*}$, adds the tuple $\left\langle U, \tau, T, I D_{A}, P K_{A}, I D_{B}, P K_{B}, h_{i}, H=h_{i} P\right\rangle$ to the list $L_{3}$ and returns $H$ to $\mathcal{A}_{I I}$.

- $\mathcal{O}_{H_{4}}\left(U, \tau, T, I D_{A}, P K_{A}, I D_{B}, P K_{B}\right)$ : To respond to this query, $\mathcal{C}$ checks whether a tuple of the form $\left\langle U, \tau, T, I D_{A}, P K_{A}, I D_{B}, P K_{B}, h_{i}^{\prime}, H^{\prime}\right\rangle$ exists in the list $L_{4}$. If so, returns $H^{\prime}$ to $\mathcal{A}_{I}$ else performs the following:

- If $I D_{A} \neq I D_{\gamma}, \mathcal{C}$ chooses $h_{i}^{\prime} \in_{R} \mathbb{Z}_{q}^{*}$, adds the tuple $\left\langle U, \tau, T, I D_{A}, P K_{A}, I D_{B}, P K_{B}, h_{i}^{\prime}, H^{\prime}=h_{i}^{\prime} P\right\rangle$ to the list $L_{4}$ and returns $H^{\prime}$ to $\mathcal{A}_{I I}$.

- If $I D_{A}=I D_{\gamma}, \mathcal{C}$ chooses $h_{i}^{\prime} \in_{R} \mathbb{Z}_{q}^{*}$, adds the tuple $\left\langle U, \tau, T, I D_{A}, P K_{A}, I D_{B}, P K_{B}, h_{i}^{\prime}, H^{\prime}=\right.$ $\left.h_{i}^{\prime} b P\right\rangle$ to the list $L_{4}$ and returns $H^{\prime}$ to $\mathcal{A}_{I I}$.

- $\mathcal{O}_{\text {ExtractPartialPrivateKey }}\left(I D_{i}\right)$ : On a request for the partial private key of a user with identity $I D_{i}$, $\mathcal{C}$ retrieves $b_{i}$ corresponding to $I D_{i}$ from list $L_{1}$ and returns $D_{i}=b_{i} s P$.

(Note: It is assumed throughout the confidentiality game, $\mathcal{A}_{I I}$ queries $\mathcal{O}_{H_{1}}$ oracle with $I D_{i}$ before querying any other oracles with $I D_{i}$ as input.)

- $\mathcal{O}_{\text {RequestPublicKey }}\left(I D_{i}\right)$ : During Phase I, the model requires the adversary $\mathcal{A}_{I I}$ and the challenger $\mathcal{C}$ to work with a signcryption from user $U_{A}$ to $U_{B}$, where $U_{A}$ and $U_{B}$ satisfy the following conditions:

- The hash of the identity $I D_{A}$ of the sender $U_{A}$ should have been queried by $\mathcal{A}_{I I}$ to $\mathcal{O}_{H_{1}}$.

- At the same time, $\mathcal{A}_{I I}$ should not know which of the identity that $\mathcal{A}_{I I}$ has queried is selected by $\mathcal{C}$ for the challenge phase.

In order to achieve the selection of identities satisfying the above conditions, we specify the Request Public Key oracle as follows:

$\mathcal{A}_{I I}$ will generate a series of user identities and send each of them to $\mathcal{C}$ and ask their corresponding public key. One of them will be chosen by $\mathcal{C}$, say the $\gamma^{\text {th }}$ distinct user identity, as target identity for the challenge phase. However, $\mathcal{C}$ will not reveal the value of $\gamma$ or $I D_{\gamma}$ to $\mathcal{A}_{I I}$. Moreover, $\mathcal{C}$ will choose $\gamma$ randomly for each game. Thus, while the target identity is one of the identities chosen by $\mathcal{A}_{I I}, \mathcal{A}_{I I}$ will not know which one is that. From now on, $I D_{\gamma}$ denotes the specific target identity selected by $\mathcal{C}$.

The oracle description and the responses by $\mathcal{C}$ are described now. $\mathcal{A}_{I I}$ produces an identity $I D_{i}$ to $\mathcal{C}$ and requests $I D_{i}$ 's public key. $\mathcal{C}$ performs the following :

- The response of $\mathcal{C}$ depends upon whether the query is $\gamma^{\text {th }}$ query or not.

Case 1: If the query is not the $\gamma^{\text {th }}$ query then $\mathcal{C}$ proceeds as follows:

* $\mathcal{C}$ checks in list $L_{K}$ whether an entry of the type $\left\langle I D_{i}, x_{i}, D_{i}, P K_{i}\right\rangle$ already exists in the list. If a tuple appears in $L_{K}, \mathcal{C}$ retrieves $P K_{i}$ from the tuple and returns it as the public key of $I D_{i}$ to $\mathcal{A}_{I I}$.

* If no matching tuple exists for $I D_{i}, \mathcal{C}$ chooses $x_{i} \in_{R} \mathbb{Z}_{q}^{*}$, generates the public key $P K_{i}=x_{i} P$ corresponding to $I D_{i}$, stores $\left\langle I D_{i}, x_{i},-, P K_{i}\right\rangle$ in $L_{K}$ and sends $P K_{i}$ to $\mathcal{A}_{I I}$.

Case 2: If the query is the $\gamma^{\text {th }}$ query then $\mathcal{C}$ proceeds as follows:

* Checks whether a tuple of the form $\left\langle I D_{i}, x_{i}, D_{i}, P K_{i}\right\rangle$, corresponding to $I D_{i}$ exists in list $L_{K}$, if it is available then $\mathcal{C}$ retrieves $P K_{i}$ from the tuple and returns it as the public key of $I D_{i}$ to $\mathcal{A}_{I I}$.

* If the query is the $\gamma^{\text {th }}$ query and no tuple corresponding to $I D_{i}$ exists in list $L_{K}, \mathcal{C}$ secretly sets $I D_{\gamma}=I D_{i}$ (The target identity) and proceeds as follows:

1. Sets $P K_{\gamma}=a P$.

2. Adds $\left\langle I D_{i},-,-, P K_{i}\right\rangle$ to the list $L_{K}$.

3. Sends $P K_{i}$ to $\mathcal{A}_{I I}$.

- $\mathcal{O}_{\text {ExtractPrivateKey }}\left(I D_{i}\right): \mathcal{A}_{I}$ produces an identity $I D_{i}$ and requests the corresponding full private key. If $I D_{i}$ 's public key has not been replaced and if $I D_{i} \neq I D_{\gamma}$, then $\mathcal{C}$ responds with the full private key $S_{i}=\left\langle x_{i}, D_{i}\right\rangle$ retrieving it from the list $L_{K}$. If $\mathcal{A}_{I I}$ has already replaced $I D_{i}$ 's public key, then $\mathcal{C}$ does not provide the corresponding private key to $\mathcal{A}_{I I}$ (Note that $\mathcal{A}_{I I}$ is allowed to replace the public key of any identity excluding $I D_{\gamma}$, thus $\mathcal{A}_{I I}$ is allowed to know the user secret value corresponding to all the identity excluding $\left.I D_{\gamma}\right) . \mathcal{A}_{I I}$ aborts if $I D_{i}=I D_{\gamma}$. 
- $\mathcal{O}_{\text {ReplacePublicKey }}\left(P K_{i}^{\prime}\right)$ : The public key $P K_{i}$ corresponding to the identity $I D_{i}$ can be replaced by another value $P K_{i}^{\prime}$ which is chosen by $\mathcal{A}_{I I}$. Before replacing, $\mathcal{C}$ checks whether $I D_{i}=I D_{\gamma}$ if so, $\mathcal{C}$ aborts the game; else, $\mathcal{C}$ updates the corresponding tuple in the list $L_{K}$ as $\left\langle I D_{i},-, D_{i}, P K_{i}^{\prime}\right\rangle$. Henceforth the current public key (i.e. replaced public key) is used by $\mathcal{C}$ for computations or responses to any queries made by $\mathcal{A}_{I I}$.

- $\mathcal{O}_{\text {SymmetricKeyGeneration }}: \mathcal{A}_{I I}$ produces a sender's identity $I D_{A}$, public key $P K_{A}$, the receiver's identity $I D_{B}$ and public key $P K_{B}$ to $\mathcal{C}$. Now, $\mathcal{C}$ computes the symmetric key $K$ and an internal state information $\omega$, stores and keeps $\omega$ secret from the view of $\mathcal{A}_{I I}$ and sends the symmetric key $K$ to $\mathcal{A}_{I I}$. It is to be noted that $\mathcal{C}$ can perform this even if $\mathcal{C}$ does not know the private key corresponding to the sender $I D_{A}$ or the receiver $I D_{B}$ because computing $K$ does not involve the private key of either the sender or receiver.

- $\mathcal{O}_{\text {KeyEncapsulation }}: \mathcal{A}_{I I}$ produces an arbitrary tag $\tau$, the sender's identity $I D_{A}$, public key $P K_{A}$, the receiver's identity $I D_{B}$ and public key $P K_{B}$ to $\mathcal{C}$. The full private key of the sender $S_{A}=\left(x_{A}, D_{A}\right)$ is obtained from the list $L_{K} \cdot \mathcal{C}$ checks whether a corresponding $\omega$ value is stored previously.

- If $\omega$ does not exist, $\mathcal{C}$ returns invalid.

- If a corresponding $\omega$ exists and $I D_{A} \neq I D_{\gamma}$, then $\mathcal{C}$ computes the encapsulation $\psi$ with $\omega$ and $\tau$ as per the actual encapsulation algorithm, and deletes $\omega$.

- If a corresponding $\omega$ exists and $I D_{A}=I D_{\gamma}$, then $\mathcal{C}$ performs the following to compute $\psi$ (It is to be noted that $\mathcal{C}$ does not know the private key corresponding to $I D_{\gamma}$, so it cooks up the encapsulation in a different way):

* Chooses $r, h_{i}, h_{i}^{\prime} \in_{R} \mathcal{Z}_{q}^{*}$ and computes $U=r P-h_{i}^{-1} h_{i}^{\prime} P K_{A}$, where $P K_{A}$ is obtained from the list $L_{K}$.

* Computes $H=h_{i} P$ and adds the tuple $\left\langle U, \tau, T, I D_{A}, P K_{A}, I D_{B}, P K_{B}, h_{i}, H\right\rangle$ to the list $L_{3}$.

* Computes $H^{\prime}=h_{i}^{\prime} P$ and adds the tuple $\left\langle U, \tau, T, I D_{A}, P K_{A}, I D_{B}, P K_{B}, h_{i}^{\prime}, H^{\prime}\right\rangle$ to the list $L_{4}$.

* Computes $W=r H+b_{i} s P$ (This is possible because $\mathcal{C}$ knows the the master private key $s$ ).

* Outputs, $\psi=\langle U, W\rangle$ as the encapsulation.

We show that, $\psi=\langle U, W\rangle$ passes the verification done by $\mathcal{A}_{I}$ to validate the encapsulation, because the equality $\hat{e}\left(P_{p u b}, Q_{A}\right) \hat{e}(U, H) \hat{e}\left(P K_{A}, H^{\prime}\right) \stackrel{?}{=} \hat{e}(P, W)$ holds.

\section{Correctness:}

$$
\begin{aligned}
\hat{e}\left(P_{p u b}, Q_{A}\right) \hat{e}(U, H) \hat{e}\left(P K_{A}, H^{\prime}\right) & =\hat{e}\left(s P, b_{i} P\right) \hat{e}\left(r P-h_{i}^{-1} h_{i}^{\prime} P K_{A}, H\right) \hat{e}\left(P K_{A}, H^{\prime}\right) \\
& =\hat{e}\left(s P, b_{i} P\right) \hat{e}(r P, H) \hat{e}\left(-h_{i}^{-1} h_{i}^{\prime} P K_{A}, H\right) \hat{e}\left(P K_{A}, H^{\prime}\right) \\
& =\hat{e}\left(s P, b_{i} P\right) \hat{e}\left(r P, h_{i} P\right) \hat{e}\left(-h_{i}^{-1} h_{i}^{\prime} P K_{A}, h_{i} P\right) \hat{e}\left(P K_{A}, h_{i}^{\prime} P\right) \\
& =\hat{e}\left(s P, b_{i} P\right) \hat{e}\left(r P, h_{i} P\right) \hat{e}\left(-h_{i}^{\prime} P K_{A}, P\right) \hat{e}\left(P K_{A}, h_{i}^{\prime} P\right) \\
& =\hat{e}\left(s P, b_{i} P\right) \hat{e}\left(r P, h_{i} P\right) \\
& =\hat{e}\left(P, r h_{i} P+b_{i} s P\right) \\
& =\hat{e}(P, W)
\end{aligned}
$$

- $\mathcal{O}_{\text {KeyDecapsulation }}: \mathcal{A}_{I I}$ produces an encapsulation $\psi$, a tag $\tau$, the sender's identity $I D_{A}$, public key $P K_{A}$, the receiver's identity $I D_{B}$ and public key $P K_{B}$ to $\mathcal{C}$. The private key of the receiver $S_{B}$ is obtained from the list $L_{K}$. It is to be noted that $\mathcal{C}$ may not be aware of the corresponding private key if the public key of $I D_{B}$ is replaced. In this case $\mathcal{C}$ obtains the private key of $I D_{B}$ from $\mathcal{A}_{I}$.

- If $I D_{B} \neq I D_{\gamma}$, then $\mathcal{C}$ computes the decapsulation of $\psi$ as per the actual decapsulation algorithm.

- If $I D_{B}=I D_{\gamma}$, then $\mathcal{C}$ computes $K$ from $\psi$ as follows:

* Searches in the list $L_{3}$ and $L_{4}$ for entries of the type $\left\langle U, \tau, T, I D_{A}, P K_{A}, I D_{B}, P K_{B}, h_{i}, H\right\rangle$ and $\left\langle U, T, \tau, I D_{A}, P K_{A}, I D_{B}, P K_{B}, h_{i}^{\prime}, H^{\prime}\right\rangle$ respectively.

* If entries $H$ and $H^{\prime}$ exist then $\mathcal{C}$ checks whether the equality $\hat{e}\left(P_{p u b}, Q_{A}\right) \hat{e}(U, H) \hat{e}\left(P K_{A}, H^{\prime}\right) \stackrel{?}{=}$ $\hat{e}(P, W)$ holds.

* If the above equality holds, then retrieves the corresponding value of $T$ from the lists $L_{3}$ and $L_{4}$ (note that both the $T$ values should be equal).

* Now, $\mathcal{C}$ checks whether a tuple of the form $\left\langle U, T, U^{\prime}=x_{B} U, I D_{B}, P K_{B}, K\right\rangle$ exists in the list $L_{2}$. If it exists then check whether $\hat{e}\left(U^{\prime}, P\right) \stackrel{?}{=} \hat{e}(U, b P)$. If the check holds then output the corresponding $K$ value as the decapsulation of $\psi$. 
Challenge: At the end of Phase $1, \mathcal{A}_{I I}$ sends to $\mathcal{C}$, a sender identity $I D_{A^{*}}$ and a receiver identity $I D_{B^{*}}$ on which $\mathcal{A}_{I I}$ wishes to be challenged. Here, the full private key of the receiver $I D_{B^{*}}$ was not queried in Phase 1. $\mathcal{C}$ aborts the game if $I D_{B^{*}} \neq I D_{\gamma}, \mathcal{C}$ performs the following to compute the challenge encapsulation $\psi^{*}$.

- Sets $U=b P$ and computes $T=\hat{e}\left(D_{B}, U\right)$.

- Chooses $K_{0} \in_{R} \mathcal{K}$, where $\mathcal{K}$ is the key space of the ICLSC-TKEM scheme

- Chooses $U^{\prime} \in_{R} \mathbb{G}_{1}$ and computes $K_{1}=H_{2}\left(U, T, U^{\prime}, I D_{B}, P K_{B}\right)$.

- Sets $\omega^{*}=\left\langle-, U, U^{\prime}, T, I D_{A}, P K_{A}, S_{A}, I D_{B}, P K_{B}\right\rangle$.

- Now, $\mathcal{C}$ chooses a bit $\delta \in_{R}\{0,1\}$ and sends $K_{\delta}$ to $\mathcal{A}_{I I}$.

- $\mathcal{A}_{I I}$ generates an arbitrary tag $\tau^{*}$ and sends it to $\mathcal{C}$.

- Chooses $h_{i}, h_{i}^{\prime} \in_{R} \mathbb{Z}_{q}^{*}$, stores the tuple $\left\langle U, \tau^{*}, T, I D_{A}, P K_{A}, I D_{B}, P K_{B}, h_{i}, H=h_{i} P\right\rangle$ to the list $L_{3}$ and $\left\langle U, \tau^{*}, T, I D_{A}, P K_{A}, I D_{B}, P K_{B}, h_{i}^{\prime}, H^{\prime}=h_{i}^{\prime} P\right\rangle$ to the list $L_{4}$.

- Since $\mathcal{C}$ knows the private key of the sender, $\mathcal{C}$ computes $W=D_{A}+h_{i} b P+h_{i}^{\prime} x_{A} P$.

$-\mathcal{C}$ now sends $\psi^{*}=\langle U, W\rangle$ to $\mathcal{A}_{I I}$.

Phase II: $\mathcal{A}_{I I}$ adaptively queries the oracles as in Phase I, consistent with the constraints for Type-II adversary. Besides this it cannot query decapsulation on $\psi^{*}$.

Guess: Since $\mathcal{A}_{I I}$ is capable of breaking the IND-ICLSC-TKEM-CCA2-II security of ICLSC-TKEM (which is assumed at the beginning of the proof), $\mathcal{A}_{I I}$ should have queried $\mathcal{O}_{H_{2}}$ with $\left(U, T, U^{\prime}, I D_{B}, P K_{B}\right)$ as inputs. it is to be noted that $U^{\prime}=x_{B} U=a b P$. Therefore, if the list $L_{2}$ has $q_{H_{2}}$ queries corresponding to the sender $I D_{A}$ and receiver $I D_{B}$, one of the $U^{\prime}$ 's among $q_{H_{2}}$ values stored in the list $L_{2}$, is the solution for the $\mathrm{CDH}$ problem instance. Now, $\mathcal{C}$ chooses one $T$ value uniformly at random from the $q_{H_{2}}$ values from the list $L_{2}$ and outputs it as the solution for the $\mathrm{CDH}$ instance.

Analysis: We now assess the probability of success of the challenger $\mathcal{C}$. The events in which $\mathcal{C}$ aborts the IND-ICLSC-TKEM-CCA2-II game are,

1. $E_{1}$ - when $\mathcal{A}_{I I}$ queries the full private key of the target identity $I D_{\gamma}$ and $\operatorname{Pr}\left[E_{1}\right]=\frac{q_{f p k}}{q_{H_{1}}}$.

2. $E_{2}$ - when $\mathcal{A}_{I I}$ requests to replace the public key of the target identity $I D_{\gamma}$ and $\operatorname{Pr}\left[E_{2}\right]=\frac{q_{r p k}}{q_{H_{1}}}$.

3. $E_{3}$ - when $\mathcal{A}_{I I}$ does not choose the target identity $I D_{\gamma}$ as the receiver during the challenge and $\operatorname{Pr}\left[E_{3}\right]=$ $\left(1-\frac{1}{q_{H_{1}}-\left(q_{f p k}+q_{r p k}\right)}\right)$.

The probability that, $\mathcal{C}$ does not abort the IND-ICLSC-TKEM-CCA2-II game is given by

$$
\left(\operatorname{Pr}\left[\neg E_{1} \wedge \neg E_{2} \wedge \neg E_{3}\right]\right)=\left(1-\frac{q_{f p k}}{q_{H_{1}}}\right)\left(1-\frac{q_{r p k}}{q_{H_{1}}}\right)\left(\frac{1}{q_{H_{1}}-\left(q_{f p k}+q_{r p k}\right)}\right)
$$

The probability that, the $U^{\prime}$ chosen randomly from $L_{2}$ by $\mathcal{C}$, being the solution to $\mathrm{CDH}$ problem is $\left(\frac{1}{q_{H_{2}}}\right)$. Therefore, the probability of $\mathcal{C}$ solving the $\mathrm{CDH}$ instance is given by,

$$
\operatorname{Pr}\left[\mathcal{C}(P, a P, b P, c P)=\hat{e}(P, P)^{a b c}\right]=\epsilon\left(1-\frac{q_{f p k}}{q_{H_{1}}}\right)\left(1-\frac{q_{r p k}}{q_{H_{1}}}\right)\left(\frac{1}{q_{H_{1}}-\left(q_{f p k}+q_{r p k}\right)}\right)\left(\frac{1}{q_{H_{2}}}\right)
$$

Since $\epsilon$ is non-negligible, the probability of $\mathcal{C}$ solving $\mathrm{CDH}$ problem is also non-negligible.

\subsection{Type-I Unforgeability}

Theorem 3. If there exists a forger $\mathcal{F}_{I}$ with an advantage $\epsilon$ against the EUF-ICLSC-TKEM-CMA-I security of the ICLSC-TKEM scheme, asking $q_{H_{i}}(i=1,2,3,4)$ hash queries to random oracles $\mathcal{O}_{H_{i}}(i=1$, 2, 3, 4), $q_{p p k}$ partial private key extract queries and $q_{f p k}$ full private key extract queries, then there exist an algorithm $\mathcal{C}$ that solves the $C D H$ problem with an advantage

$$
\epsilon^{\prime} \geq\left(\epsilon-\frac{1}{2^{\kappa-1}}\right)\left(1-\frac{q_{p p k}}{q_{H_{1}}}\right)\left(1-\frac{q_{f p k}}{q_{H_{1}}}\right)\left(\frac{1}{q_{H_{1}}-\left(q_{p p k}+q_{f p k}\right)}\right)
$$


Proof: A challenger $\mathcal{C}$ is challenged with an instance of the $\mathrm{CDH}$ problem say $\langle P, a P, b P\rangle \in \mathbb{G}_{1}$. Let $\mathcal{F}_{I}$ be a forger who is capable of breaking the EUF-ICLSC-TKEM-CMA-I security of the ICLSC-TKEM scheme. $\mathcal{C}$ can make use of $\mathcal{F}_{I}$ to compute the solution $a b P$ of the $\mathrm{CDH}$ instance by playing the following interactive game with $\mathcal{F}_{I}$.

Setup: $\mathcal{C}$ sets the master public key $P_{p u b}$ as $a P$, designs the hash functions $H_{i}(i=1$ to 4$)$ as random oracles $\mathcal{O}_{H_{i}}(i=1$ to 4$)$ respectively. In order to maintain the consistency between the responses to the hash queries, $\mathcal{C}$ maintains lists $L_{i}(i=1$ to 4$)$ and to maintain the list of issued private keys and public keys, $\mathcal{C}$ maintains a list $L_{K}$. $\mathcal{C}$ gives the public parameters params to $\mathcal{F}_{I}$.

Training Phase: $\mathcal{F}_{I}$ performs a series of polynomially bounded number of queries in an adaptive fashion in this phase. The oracles and queries allowed are described below.

- All the oracles are identical to that of the $\mathcal{O}_{H_{1}}, \mathcal{O}_{H_{2}}, \mathcal{O}_{H_{3}}, \mathcal{O}_{H_{4}}, \mathcal{O}_{\text {ExtractPartialPrivateKey }}, \mathcal{O}_{\text {Request }}$ PublicKey $, \mathcal{O}_{\text {ExtractPrivateKey }}, \mathcal{O}_{\text {ReplacePublicKey }}, \mathcal{O}_{\text {SymmetricKeyGeneration }}, \mathcal{O}_{\text {KeyEncapsulation }}$ and $\mathcal{O}_{\text {KeyDecapsulation }}$ oracles in the IND-ICLSC-TKEM-CCA2-I game.

Forgery: At the end of the Training Phase (which is decided by $\mathcal{F}_{I}$ ), $\mathcal{F}_{I}$ sends to $\mathcal{C}$ an encapsulation $\left\langle\tau^{*}, \psi^{*}=\langle U, W\rangle, I D_{\gamma}, I D_{B}\right\rangle$, where $I D_{\gamma}$ is the sender identity and $I D_{B}$ is the receiver identity. It is to be noted that the partial private key of the sender $I D_{\gamma}$ was not queried and the public key of $I D_{\gamma}$ could be replaced during the Training Phase. In addition, $\psi^{*}$ should not be the response for any key encapsulation queries by $\mathcal{F}_{I}$ during the Training Phase. If $\psi^{*}$ is generated with the above restrictions, then $\mathcal{C}$ can obtain the solution for the $\mathrm{CDH}$ instance by performing the following steps.

- Retrieves the tuple $\left\langle U, \tau^{*}, T, I D_{\gamma}, P K_{\gamma}, I D_{B}, P K_{B}, h_{i}, H=h_{i} P\right\rangle$ from the list $L_{3}$ and $\left\langle U, \tau^{*}, T, I D_{\gamma}\right.$, $\left.P K_{\gamma}, I D_{B}, P K_{B}, h_{i}, H^{\prime}=h_{i}^{\prime} P\right\rangle$ from the list $L_{4}$.

- Output $W-h_{i} U-x_{\gamma} h_{i}^{\prime} P=a b P$ as the solution to the CDH problem instance.

\section{Correctness:}

$$
\begin{aligned}
W-h_{i} U-x_{\gamma} h_{i}^{\prime} P & =D_{\gamma}+r H+x_{\gamma} H^{\prime}-h_{i} U-x_{\gamma} h_{i}^{\prime} P \\
& =D_{\gamma}+r H+x_{\gamma} H^{\prime}-r h_{i} P-x_{\gamma} H^{\prime} \\
& =D_{\gamma}+r H-r H \\
& =D_{\gamma} \\
& =a b P
\end{aligned}
$$

Analysis: We now assess the probability of success of the challenger $\mathcal{C}$. The events in which $\mathcal{C}$ aborts the EUF-ICLSC-TKEM-CMA-I game are $E_{1}, E_{2}$ and $E_{3}$. (Events $E_{1}, E_{2}$ and $E_{3}$ are same as in IND-ICLSCTKEM-CCA2-I proof). The probability that, $\mathcal{C}$ does not abort the EUF-ICLSC-TKEM-CMA-I game is given by

$$
\left(\operatorname{Pr}\left[\neg E_{1} \wedge \neg E_{2} \wedge \neg E_{3}\right]\right)=\left(1-\frac{q_{p p k}}{q_{H_{1}}}\right)\left(1-\frac{q_{f p k}}{q_{H_{1}}}\right)\left(\frac{1}{q_{H_{1}}-\left(q_{p p k}+q_{f p k}\right)}\right)
$$

The probability of $\mathcal{F}_{I}$ guessing the hash values corresponding to $H_{3}$ and $H_{4}$ oracles is $\frac{1}{2^{\kappa}}+\frac{1}{2^{\kappa}}=\frac{1}{2^{\kappa-1}}$. The probability with which $\mathcal{C}$ solves the $\mathrm{CDH}$ problem is

$$
\epsilon^{\prime} \geq\left(\epsilon-\frac{1}{2^{\kappa-1}}\right)\left(1-\frac{q_{p p k}}{q_{H_{1}}}\right)\left(1-\frac{q_{f p k}}{q_{H_{1}}}\right)\left(\frac{1}{q_{H_{1}}-\left(q_{p p k}+q_{f p k}\right)}\right)
$$

Since $\epsilon$ is non-negligible, the probability of $\mathcal{C}$ solving $\mathrm{CDH}$ problem is also non-negligible.

\subsection{Type-II Unforgeability}

Theorem 4. If there exists a forger $\mathcal{F}_{I}$ with an advantage $\epsilon$ against the EUF-ICLSC-TKEM-CMA-I security of the ICLSC-TKEM scheme, asking $q_{H_{i}}(i=1,2,3,4)$ hash queries to random oracles $\mathcal{O}_{H_{i}}(i=1$, 2, 3, 4), $q_{f p k}$ full private key extract queries and $q_{f p k}$ replace public key queries, then there exist an algorithm $\mathcal{C}$ that solves the $C D H$ problem with an advantage

$$
\epsilon^{\prime} \geq\left(\epsilon-\frac{1}{2^{\kappa-1}}\right)\left(1-\frac{q_{f p k}}{q_{H_{1}}}\right)\left(1-\frac{q_{r p k}}{q_{H_{1}}}\right)\left(\frac{1}{q_{H_{1}}-\left(q_{f p k}+q_{r p k}\right)}\right)
$$


Proof: A challenger $\mathcal{C}$ is challenged with an instance of the $\mathrm{CDH}$ problem say $\langle P, a P, b P\rangle \in \mathbb{G}_{1}$. Let $\mathcal{F}_{I I}$ be a forger who is capable of breaking the EUF-ICLSC-TKEM-CMA-II security of the ICLSC-TKEM scheme. $\mathcal{C}$ can make use of $\mathcal{F}_{I I}$ to compute the solution $a b P$ of the $\mathrm{CDH}$ instance by playing the following interactive game with $\mathcal{F}_{I I}$.

Setup: $\mathcal{C}$ chooses $s \in_{R} \mathbb{Z}_{q}^{*}$ and sets the master public key $P_{p u b}=s P$, designs the hash functions $H_{i}(i=1$ to 4) as random oracles $\mathcal{O}_{H_{i}}(i=1$ to 4$)$ respectively. In order to maintain the consistency between the responses to the hash queries, $\mathcal{C}$ maintains lists $L_{i}(i=1$ to 4$)$ and to maintain the list of issued private keys and public keys, $\mathcal{C}$ maintains a list $L_{K}$. $\mathcal{C}$ gives the public parameters params and the master private key $s$ to $\mathcal{F}_{I I}$.

Training Phase: $\mathcal{F}_{I I}$ performs a series of polynomially bounded number of queries in an adaptive fashion in this phase. The oracles and queries allowed are described below.

- All the oracles are identical to that of the $\mathcal{O}_{H_{1}}, \mathcal{O}_{H_{2}}, \mathcal{O}_{H_{3}}, \mathcal{O}_{H_{4}}, \mathcal{O}_{\text {ExtractPartialPrivateKey }}, \mathcal{O}_{\text {Request }}$ PublicKey $, \mathcal{O}_{\text {ExtractPrivateKey }}, \mathcal{O}_{\text {ReplacePublicKey }}, \mathcal{O}_{\text {SymmetricKeyGeneration }}, \mathcal{O}_{\text {KeyEncapsulation }}$ and $\mathcal{O}_{\text {KeyDecapsulation }}$ oracles in the IND-ICLSC-TKEM-CCA2-II game.

Forgery: At the end of the Training Phase (which is decided by $\mathcal{F}_{I I}$ ), $\mathcal{F}_{I I}$ sends to $\mathcal{C}$ an encapsulation $\left\langle\tau^{*}, \psi^{*}=\langle U, W\rangle, I D_{\gamma}, I D_{B}\right\rangle$, where $I D_{\gamma}$ is the sender identity and $I D_{B}$ is the receiver identity. It is to be noted that the public key of the sender $I D_{\gamma}$ was not replaced during the Training Phase. In addition, $\psi^{*}$ should not be the response for any key encapsulation queries by $\mathcal{F}_{I}$ during the Training Phase. If $\psi^{*}$ is generated with the above restrictions, then $\mathcal{C}$ can obtain the solution for the CDH instance by performing the following steps.

- Retrieves the tuple $\left\langle U, \tau^{*}, T, I D_{\gamma}, P K_{\gamma}, I D_{B}, P K_{B}, h_{i}, H=h_{i} P\right\rangle$ from the list $L_{3}$ and $\left\langle U, \tau^{*}, T, I D_{\gamma}\right.$, $\left.P K_{\gamma}, I D_{B}, P K_{B}, h_{i}, H^{\prime}=h_{i}^{\prime} P\right\rangle$ from the list $L_{4}$.

- Output $h_{i}^{\prime}-1\left(W-s b_{\gamma} P-h_{i} U\right)=a b P$ as the solution to the CDH problem instance.

\section{Correctness:}

$$
\begin{aligned}
h_{i}^{\prime}-1\left(W-s b_{\gamma} P-h_{i} U\right) & =h_{i}^{\prime}-1\left(D_{\gamma}+r H+a h_{i}^{\prime} b P-s b_{\gamma} P-h_{i} U\right) \\
& =h_{i}^{\prime}-1\left(b_{\gamma} s P+r h_{i} P+a h_{i}^{\prime} b P-s b_{\gamma} P-r h_{i} P\right) \\
& =h_{i}^{\prime}-1\left(a h_{i}^{\prime} b P\right) \\
& =a b P
\end{aligned}
$$

Analysis: We now assess the probability of success of the challenger $\mathcal{C}$. The events in which $\mathcal{C}$ aborts the EUF-ICLSC-TKEM-CMA-II game are $E_{1}, E_{2}$ and $E_{3}$. (Events $E_{1}, E_{2}$ and $E_{3}$ are same as in IND-ICLSCTKEM-CCA2-II proof). The probability that, $\mathcal{C}$ does not abort the EUF-ICLSC-TKEM-CMA-II game is given by

$$
\left(\operatorname{Pr}\left[\neg E_{1} \wedge \neg E_{2} \wedge \neg E_{3}\right]\right)=\left(1-\frac{q_{f p k}}{q_{H_{1}}}\right)\left(1-\frac{q_{r p k}}{q_{H_{1}}}\right)\left(\frac{1}{q_{H_{1}}-\left(q_{f p k}+q_{r p k}\right)}\right)
$$

The probability of $\mathcal{F}_{I I}$ guessing the hash values corresponding to $H_{3}$ and $H_{4}$ oracles is $\frac{1}{2^{\kappa}}+\frac{1}{2^{\kappa}}=\frac{1}{2^{\kappa-1}}$. The probability with which $\mathcal{C}$ solves the $\mathrm{CDH}$ problem is

$$
\epsilon^{\prime} \geq\left(\epsilon-\frac{1}{2^{\kappa-1}}\right)\left(1-\frac{q_{f p k}}{q_{H_{1}}}\right)\left(1-\frac{q_{r p k}}{q_{H_{1}}}\right)\left(\frac{1}{q_{H_{1}}-\left(q_{f p k}+q_{r p k}\right)}\right)
$$

Since $\epsilon$ is non-negligible, the probability of $\mathcal{C}$ solving $\mathrm{CDH}$ problem is also non-negligible.

\section{Conclusion}

In this paper, we have showed that the only existing CL-KEM [13] proved in the standard model is not Type-I CCA secure and the only existing certificateless hybrid signcryption scheme of Fagen Li et al.'s [15] is existentially forgeable with respect to both Type-I and Type-II forgers. We have also proposed an improved certificateless hybrid signcryption scheme with the proper binding, that provides adequate security to the scheme. We have proved the improved certificateless hybrid signcryption scheme in the random oracle model. 


\section{References}

1. Sattam S. Al-Riyami and Kenneth G. Paterson. Certificateless public key cryptography. In Advances in Cryptology - ASIACRYPT 2003, volume 2894 of Lecture Notes in Computer Science, pages 452-473. Springer, 2003.

2. Jee Hea An, Yevgeniy Dodis, and Tal Rabin. On the security of joint signature and encryption. In Advances in Cryptology - EUROCRYPT 2002, volume 2332 of Lecture Notes in Computer Science, pages 83-107. Springer, 2002.

3. Joonsang Baek, Ron Steinfeld, and Yuliang Zheng. Formal proofs for the security of signcryption. In Public Key Cryptography - PKC 2002, volume 2274 of Lecture Notes in Computer Science, pages 80-98. Springer, 2002.

4. Manuel Barbosa and Pooya Farshim. Certificateless signcryption. In ACM Symposium on Information, Computer and Communications Security - ASIACCS 2008, pages 369-372. ACM, 2008.

5. Paulo S. L. M. Barreto, Benoît Libert, Noel McCullagh, and Jean-Jacques Quisquater. Efficient and provablysecure identity-based signatures and signcryption from bilinear maps. In Advances in Cryptology - ASIACRYPT 2005, volume 3788 of Lecture Notes in Computer Science, pages 515-532. Springer, 2005.

6. Kamel Bentahar, Pooya Farshim, John Malone-Lee, and Nigel P. Smart. Generic constructions of identity-based and certificateless kems. J. Cryptology, 21(2):178-199, 2008.

7. Xavier Boyen. Multipurpose identity-based signcryption (a swiss army knife for identity-based cryptography). In Advances in Cryptology - CRYPTO 2003, volume 2729 of Lecture Notes in Computer Science, pages 383-399. Springer, 2003.

8. Liqun Chen and John Malone-Lee. Improved identity-based signcryption. In Public Key Cryptography - PKC 2005, volume 3386 of Lecture Notes in Computer Science, pages 362-379. Springer, 2005.

9. Sherman S. M. Chow, Siu-Ming Yiu, Lucas Chi Kwong Hui, and K. P. Chow. Efficient forward and provably secure id-based signcryption scheme with public verifiability and public ciphertext authenticity. In ICISC, volume 2971 of Lecture Notes in Computer Science, pages 352-369. Springer, 2003.

10. Ronald Cramer and Victor Shoup. Design and analysis of practical public-key encryption schemes secure against adaptive chosen ciphertext attack. SIAM Journal on Computing, 33(1):167-226, 2003.

11. Alexander W. Dent. Hybrid signcryption schemes with insider security. In Information Security and Privacy ACISP 2005, volume 3574 of Lecture Notes in Computer Science, pages 253-266. Springer, 2005.

12. Yevgeniy Dodis, Michael J. Freedman, Stanislaw Jarecki, and Shabsi Walfish. Versatile padding schemes for joint signature and encryption. In ACM Conference on Computer and Communications Security - CCS 2004, pages 344-353. ACM, 2004.

13. Juan González Nieto Georg Lippold, Colin Boyd. Efficient certificateless kem in the standard model. Cryptology ePrint Archive, Report 2009/451 (Extended abstract of the paper accepted in ICISC-09), 2009. http://eprint.iacr.org/.

14. Qiong Huang and Duncan S. Wong. Generic certificateless key encapsulation mechanism. In ACISP 2007, volume 4586 of Lecture Notes in Computer Science, pages 215-229. Springer, 2007.

15. Fagen Li, Masaaki Shirase, and Tsuyoshi Takagi. Certificateless hybrid signcryption. In Information Security Practice and Experience - ISPEC 2009, volume 5451 of Lecture Notes in Computer Science, pages 112-123. Springer, 2009.

16. Benoît Libert and Jean-Jacques Quisquater. Efficient signcryption with key privacy from gap diffie-hellman groups. In Public Key Cryptography - PKC 2004, volume 2947 of Lecture Notes in Computer Science, pages 187-200. Springer, 2004.

17. Benot Libert and Jean-Jacques Quisquater. A new identity based signcryption scheme from pairings. In In IEEE Information Theory Workshop, pages 155-158, 2003.

18. John Malone-Lee. Identity-based signcryption. Cryptology ePrint Archive, Report 2002/098, 2002.

19. John Malone-Lee and Wenbo Mao. Two birds one stone: Signcryption using rsa. In Topics in Cryptology CT-RSA 2003, volume 2612 of Lecture Notes in Computer Science, pages 211-225. Springer, 2003.

20. S. Sharmila Deva Selvi, S. Sree Vivek, Deepanshu Shukla, and C. Pandu Rangan. Efficient and provably secure certificateless multi-receiver signcryption. In Provable Security, ProvSec - 2008, volume 5324 of Lecture Notes in Computer Science, pages 52-67. Springer, 2008.

21. Adi Shamir. Identity-based cryptosystems and signature schemes. In Advances in Cryptology, CRYPTO - 1984, volume 196 of Lecture Notes in Computer Science, pages 47-53. Springer, 1984.

22. Victor Shoup. Oaep reconsidered. In Advances in Cryptology - CRYPTO 2001, volume 2139 of Lecture Notes in Computer Science, pages 239-259. Springer, 2001.

23. T.E.Bjørstad. Provable security of signcryption. In Masters Thesis, http://www.nwo.no/tor/pdf/mscthesis.pdf. Norwegian University of Technology and Science, 2005.

24. Brent Waters. Efficient identity-based encryption without random oracles. In Advances in Cryptology - EUROCRYPT 2005, volume 3494 of Lecture Notes in Computer Science, pages 114-127. Springer, 2005.

25. Yuliang Zheng. Digital signcryption or how to achieve cost(signature \& encryption) $<<\operatorname{cost}($ signature) + cost(encryption). In Advances in Cryptology, CRYPTO - 199\%, volume 1294 of Lecture Notes in Computer Science, pages 165-179. Springer, 1997. 